\title{
A mathematical model in three-dimensional piezoelectric continuum to predict non-linear responses of piezoceramic materials
}

\author{
M K Samal $^{1 *}$, P Seshu ${ }^{2}$, U von Wagner ${ }^{4}$, P Hagedorn ${ }^{3}$, B K Dutta ${ }^{1}$, and H S Kushwaha ${ }^{1}$ \\ ${ }^{1}$ Reactor Safety Division, Bhabha Atomic Research Centre, Trombay, Mumbai, India \\ ${ }^{2}$ Department of Mechanical Engineering, Indian Institute of Technology Bombay, Mumbai, India \\ ${ }^{3}$ Department of Applied Mechanics, Technical University of Darmstadt, Darmstadt, Germany \\ ${ }^{4}$ Department of Applied Mechanics, Technical University of Berlin, Berlin, Germany
}

The manuscript was received on 10 January 2008 and was accepted after revision for publication on 28 April 2008.

DOI: 10.1243/09544062JMES1002

\begin{abstract}
It has been experimentally observed that the piezoceramic materials exhibit different types of non-linearities under different combinations of electrical and mechanical fields. When excited near resonance in the presence of weak electric fields, they exhibit typical non-linearities similar to a Duffing oscillator such as jump phenomena and the presence of superharmonics in the response spectra. In this work, these non-linearities have been modelled for a generalized three-dimensional piezoelectric continuum using higher-order quadratic and cubic terms in the electric enthalpy density function and the virtual work. The identification of the parameters of the model requires a closed form solution for non-linear response of a simplified geometry. A simple proportional damping formulation has been used in the model. Experiments have been conducted on rectangular and cylindrical geometries of piezoceramic PIC 181 at different magnitudes of applied electric fields and results have been compared with those of simulation.
\end{abstract}

Keywords: analytical solution, piezoceramic, non-linear modelling, perturbation method

\section{INTRODUCTION}

Day by day, the usage of piezoceramic materials is increasing and these materials are widely applied in many different areas of modern technology, for instance, aerospace structures [1]. Prediction of performance of the structures with piezoelectric sensors and actuators (for various control applications such as active shape, vibration, noise control, etc.) requires a thorough understanding of the piezoelectric material behaviour at different applied electrical and mechanical fields. Though the non-linearities under strong electric fields such as dielectric and butterfly hysteresis have drawn the attention of many authors recently [2-7], the modelling of non-linear behaviour of piezoceramics under weak electric fields is limited in literature. With a number of piezoelectric finite-element

* Corresponding author: Reactor Safety Division, Bhabha Atomic Research Centre, Hall 7, Trombay, Mumbai 400085, Maharashtra, India.email:mksamal@barc.gov.in
(FE) models existing in the literature to cope with complicated boundary problems of smart structures, it is very important to develop a simple procedure to obtain analytical solutions for a few simple geometries for verification of finite-element formulations and also to gain confidence in the procedure. Moreover, it is also necessary to develop analytical solutions to determine various material parameters including those of the non-linear model to be used in the finiteelement analysis. The coupling of mechanical and electrical properties of piezoelectric materials complicates the analytical solution. Piezoelectric plates comprising orthotropic or anisotropic layers are usually analysed using laminated composite plate theory. For the analytical solution of these plates, Pagano [8] first presented the exact solution of a laminated plate under cylindrical bending. Ray et al. [9-11] and Heyliger and Brooks [12] extended this methodology to develop exact solutions for the piezoelectric laminated plates using Eshelby-Stroh formalism. Later, considering thermal effect, Xu et al. [13] presented a three-dimensional analysis for the coupled 
thermo-electro-elastic response of multi-layered hybrid composite plates with four simply supported edges by using the state space method. Dube et al. [14] developed a series solution method for a single-layer thermo-electro-elastic plate subjected to cylindrical bending using the approach of Pagano [8].

To solve the dynamic problem of the piezoelectric array elements, several simplified solutions were tried for modelling the linear piezoelectric behaviour in one-, two-, and three-dimensional domains. Ray et al. [15] presented an exact solution for analysing the dynamics of composite plates with piezoelectric layers bonded to the top and bottom surfaces. Later, Ray [16] also presented the closed form solution for the optimal control of flexural vibration of a simply supported symmetric laminated plate. Wolf and Gottlieb [17] derived the non-linear equations of motion for a cantilever beam covered by piezoelectric ceramic material (PZT) layers in symmetric and asymmetric configurations. By defining a non-linear enthalpy function with non-linear strain and applying the Hamilton's principle, they derived the equations of motion and solved by perturbation analysis and multiple scales method.

Beige and Schmidt [18] modelled the nonlinearities under weak electric fields using higherorder quadratic and cubic elastic terms in the electric enthalpy function. von Wagner and Hagedorn [19] studied the non-linearity in a piezobeam system. Later von Wagner [20] and Neumann [21] predicted the amplitude of displacement and current responses of piezorods of different diameters using a onedimensional non-linear formulation. Neumann [21] used the non-linear damping formulation to derive the virtual work done by the dissipative damping forces. Samal et al. [22] presented a generalized threedimensional formulation for modelling the non-linear behaviour of piezoelectric materials under weak electric fields and it was used to derive a three-dimensional finite-element model. The application of the formulation was demonstrated in Samal et al. [23] using different types of examples. The parameter identification procedure used by von Wagner [20] and Neumann [21] is restricted to those of one-dimensional continuum. In order to predict the non-linear response of actual three-dimensional piezoelectric systems, the material properties of the three-dimensional piezoelectric continuum are required. This requires a closed form solution for a simple geometry. In this paper, such closed form solutions for predicting the displacement and current responses of simple rectangular and cylindrical geometries have been developed using a simple proportional damping formulation for the material damping.

In an earlier work, Samal et al. [24] have derived a closed form solution for a rectangular piezoceramic plate of a soft piezoceramic PIC 255. However, a non-linear damping formulation was used in the above model that requires identification of a large number of non-linear model parameters, which is very time-consuming, complicated, and cumbersome. On the other hand, Usher and Sim [25], von Wagner [20], Wang et al. [26], etc. demonstrated that the simple proportional damping theory satisfactorily predicts the response of wide varieties of piezoceramics. Hence, the objective of this work is to develop an efficient non-linear model using a simple damping theory, so that the electric field dependent damping coefficient can be evaluated easily from experimental measurements and at the same time both the mechanical and electrical responses of the piezoceramic are satisfactorily predicted. Another important aspect of this model is the use of an approach where the numerical shape functions (obtained from a prior FE analysis) can be used in place of analytical shape functions in the Rayleigh-Ritz discretization. This approach is especially helpful for geometries where the evaluation of analytical shape functions is difficult. Experiments have been conducted on plate and cylindrical geometries of a PIC 181 material (which is a hard piezoceramic) and the predicted results have been compared with those of the experiments.

\section{GENERALIZED NON-LINEAR CONSTITUTIVE EQUATION OF THE PIEZOELECTRIC CONTINUUM}

The electric enthalpy density function $H$ is generally used to derive the governing equations of the coupled piezoelectric continuum and for the linear piezoelectric behaviour, it is given as (IEEE standard [27])

$$
H=\frac{1}{2} C_{i j k l}^{\mathrm{E}} S_{i j} S_{k l}-e_{k i j} E_{k} S_{i j}-\frac{1}{2} \varepsilon_{i j}^{\mathrm{S}} \boldsymbol{E}_{i} \boldsymbol{E}_{j}
$$

where $C_{i j k l}^{\mathrm{E}}$ is the fourth-order elastic tensor under constant electric field, $e_{k i j}$ is the third-order piezoelectric tensor, $\varepsilon_{i j}^{S}$ is the second-order dielectric tensor, $S_{i j}$ is the strain tensor, and $\boldsymbol{E}_{i}$ is the electric field vector. The stress tensor $T_{i j}$ and the electric displacement vector $\boldsymbol{D}_{i}$ can be derived from $H$ using the expressions $T_{i j}=\partial H / \partial S_{i j}$ and $D_{i}=-\partial H / \partial E_{i}$, respectively. It can be easily seen that the expressions for $T_{i j}$ and $D_{i}$ are coupled with the secondary field variables $S_{i j}$ (strain tensor) and $\boldsymbol{E}_{i}$ (electric field vector), respectively. The strain tensor, $S_{i j}$ is expressed in terms of mechanical displacement vector $\boldsymbol{u}_{i}$ as $S_{i j}=\left(\boldsymbol{u}_{i, j}+\boldsymbol{u}_{j, i}\right) / 2$ and $\boldsymbol{E}_{i}$ is expressed in terms of $\phi$ as $\boldsymbol{E}_{i}=-\phi_{, i}$. Keeping in mind the fact that stress and strain tensor are symmetric, $H$ can be simplified and written in terms of second order tensors as $[\mathbf{2 8}]$

$$
H=\frac{1}{2} C_{i j}^{\mathrm{E}} S_{i} S_{j}-e_{i j} \boldsymbol{E}_{i} S_{j}-\frac{1}{2} \varepsilon_{i j}^{\mathrm{S}} \boldsymbol{E}_{i} \boldsymbol{E}_{j}
$$


In order to model the non-linearities in a coupled piezoelectric medium, the linear electric enthalpy density function is modified in the following way. It was experimentally observed $[\mathbf{1 9}, \mathbf{2 0}]$ that second- and third-order harmonics were present in the response spectra of PZT piezoceramic plates and rod geometries. Hence, the generalized expression for non-linear electric enthalpy density function can be written as [22]

$$
\begin{aligned}
H_{\mathrm{non}-\mathrm{lin}}= & \frac{1}{2} C_{i j}^{\mathrm{E}} S_{i} S_{j}-e_{m i} \boldsymbol{E}_{m} S_{i}-\frac{1}{2} \varepsilon_{m n}^{\mathrm{S}} \boldsymbol{E}_{m} \boldsymbol{E}_{n} \\
& +\frac{1}{3} C_{i j k}^{\mathrm{E}} S_{i} S_{j} S_{k}+\frac{1}{4} C_{i j k l}^{\mathrm{E}} S_{i} S_{j} S_{k} S_{l} \\
& -\frac{1}{3} \varepsilon_{m n o}^{\mathrm{S}} \boldsymbol{E}_{m} \boldsymbol{E}_{n} \boldsymbol{E}_{o}-\frac{1}{4} \varepsilon_{m n o p}^{\mathrm{S}} \boldsymbol{E}_{m} \boldsymbol{E}_{n} \boldsymbol{E}_{o} \boldsymbol{E}_{p} \\
& -\frac{1}{2} e_{m i j} \boldsymbol{E}_{m} S_{i} S_{j}-\frac{1}{2} e_{m n i}^{*} \boldsymbol{E}_{m} \boldsymbol{E}_{n} S_{i} \\
& -\frac{1}{3} e_{m i j k}^{* *} \boldsymbol{E}_{m} S_{i} S_{j} S_{k}-\frac{1}{4} e_{m n i j}^{* * *} \boldsymbol{E}_{m} \boldsymbol{E}_{n} S_{i} S_{j} \\
& -\frac{1}{3} e_{m n o i}^{* * *} \boldsymbol{E}_{m} \boldsymbol{E}_{n} \boldsymbol{E}_{o} S_{i}
\end{aligned}
$$

where the coefficients with 3 or 4 indices are higherorder cubic and quadratic coefficients, respectively. A matrix expression for $H_{\text {non-lin }}$ can be obtained from equation (3) as follows

$$
\begin{aligned}
H_{\text {non-lin }}= & \frac{1}{2}\{\boldsymbol{S}\}^{\mathrm{T}}[\mathbf{C}]\{\boldsymbol{S}\}-\{\boldsymbol{E}\}^{\mathrm{T}}[\mathbf{d}][\mathbf{C}]\{\boldsymbol{S}\} \\
& -\frac{1}{2}\{\boldsymbol{E}\}^{\mathrm{T}}\left[\left[\boldsymbol{\varepsilon}^{\mathrm{T}}\right]-[\mathbf{d}][\mathbf{C}][\mathbf{d}]^{\mathrm{T}}\right]\{\boldsymbol{E}\} \\
& +\frac{1}{3}\{\boldsymbol{S}\}^{\mathrm{T}}\left[\mathbf{C}_{1}\right]\left\{\boldsymbol{S}^{2}\right\}+\frac{1}{4}\left\{\boldsymbol{S}^{2}\right\}^{\mathrm{T}}\left[\mathbf{C}_{21}\right]\left\{\boldsymbol{S}^{2}\right\} \\
& +\frac{1}{4}\{\boldsymbol{S}\}^{\mathrm{T}}\left[\mathbf{C}_{22}\right]\left\{\boldsymbol{S}^{3}\right\}-\frac{1}{2}\{\boldsymbol{E}\}^{\mathrm{T}}\left[\boldsymbol{\gamma}_{11}\right]\left\{\boldsymbol{S}^{2}\right\} \\
& -\frac{1}{2}\left\{\boldsymbol{E}^{2}\right\}^{\mathrm{T}}\left[\gamma_{12}\right]\{\boldsymbol{S}\}-\frac{1}{3}\{\boldsymbol{E}\}^{\mathrm{T}}\left[\boldsymbol{v}_{1}\right]\left\{\boldsymbol{E}^{2}\right\} \\
& -\frac{1}{3}\{\boldsymbol{E}\}^{\mathrm{T}}\left[\boldsymbol{\gamma}_{21}\right]\left\{\boldsymbol{S}^{3}\right\}-\frac{1}{2}\left\{\boldsymbol{E}^{2}\right\}^{\mathrm{T}}\left[\boldsymbol{\gamma}_{22}\right]\left\{\boldsymbol{S}^{2}\right\} \\
& -\frac{1}{3}\left\{\boldsymbol{E}^{3}\right\}^{\mathrm{T}}\left[\gamma_{23}\right]\{\boldsymbol{S}\}-\frac{1}{4}\{\boldsymbol{E}\}^{\mathrm{T}}\left[\boldsymbol{v}_{21}\right]\left\{\boldsymbol{E}^{3}\right\} \\
& -\frac{1}{4}\left\{\boldsymbol{E}^{2}\right\}^{\mathrm{T}}\left[\boldsymbol{v}_{22}\right]\left\{\boldsymbol{E}^{2}\right\}
\end{aligned}
$$

where $\{\boldsymbol{S}\}$ is the strain vector, $[\mathbf{C}]$ is the linear elasticity matrix, $[\mathbf{d}]$ is the linear piezoelectric coefficient matrix, $\{\boldsymbol{E}\}$ is the electric field vector, $\left[\boldsymbol{\varepsilon}^{\mathrm{T}}\right]$ is the dielectric coefficient matrix, $\left[\mathbf{C}_{1}\right]$ is the quadratic elasticity matrix, and $\left[\mathbf{C}_{21}\right]$ and $\left[\mathbf{C}_{22}\right]$ are the cubic elasticity matrices at constant electric field. The superscript $\mathrm{E}$ and $\mathrm{S}$ (for the matrices at constant electric field and constant strain) are omitted for clarity. The terms $\left[\gamma_{11}\right]$ and $\left[\gamma_{12}\right]$ are quadratic piezoelectric matrices, $\left[\gamma_{21}\right],\left[\gamma_{22}\right]$, and $\left[\gamma_{23}\right]$ are cubic piezoelectric matrices, $\left[\boldsymbol{v}_{0}\right]=\left[\left[\boldsymbol{\varepsilon}^{\mathrm{T}}\right]-[\mathbf{d}][\mathbf{C}][\mathbf{d}]^{\mathrm{T}}\right],\left[\boldsymbol{v}_{1}\right],\left[\boldsymbol{v}_{21}\right]$, and $\left[\boldsymbol{v}_{22}\right]$ are linear, quadratic, and cubic dielectric matrices at constant strain field, S, respectively. The vectors with superscripts 2 and 3 are defined as the vectors with square and cube of individual terms of the vectors. The first three terms of $H_{\text {non-lin }}$ correspond to the linear electric enthalpy density function. Using this expression of $H_{\text {non-lin }}$ and the virtual work done by damping forces (described later in this paper), the closed form solutions for simple rectangular plate and cylindrical geometries have been derived in the next section using the Hamilton's principle.

\section{HAMILTON'S PRINCIPLE}

The dynamic equations of a piezoelectric continuum can be derived from the Hamilton's principle, in which the Lagrangian and the virtual work is properly adapted to include the electrical, mechanical as well as the coupled electro-mechanical terms. The potential energy density of a piezoelectric material includes contributions from the strain energy and from the electrostatic energy. The Hamilton's principle can be written as

$$
\delta \int_{t_{0}}^{t_{1}}\left(\int_{V} L \mathrm{~d} V\right) \mathrm{d} t+\int_{t_{0}}^{t_{1}} \delta W \mathrm{~d} t=0
$$

where $t_{0}$ and $t_{1}$ define the time interval, $L$ is the Lagrangian and defined in terms of the kinetic energy density $T_{\text {ke }}$, electrical enthalpy density $H$ as $L=$ $\left(T_{\mathrm{ke}}-H\right)$, and $\delta W$ is the virtual work done by external mechanical and electrical forces. The kinetic energy is given as

$$
T_{\mathrm{ke}}=\frac{1}{2} \rho\{\dot{\boldsymbol{u}}\}^{\mathrm{T}}\{\dot{\boldsymbol{u}}\}
$$

where $\rho$ is the mass density, $\{u\}$ is the generalized displacement field and $\{\dot{\boldsymbol{u}}\}$ is the generalized velocity field.

The non-linear electric enthalpy density $H_{\text {non-lin }}$ is given in equation (4). The virtual work done by the external mechanical forces $F$ and the applied electric charges $Q$ for an arbitrary variation of the displacement field $\{\delta u\}$ and electrical potential $\{\delta \phi\}$ both satisfying the essential boundary conditions (i.e. $\{\delta u\}=0$ on surface $A_{s 3}$ and $\{\delta \phi\}=0$ on surface $\left.A_{s 4}\right)$ can be written as

$$
\begin{aligned}
\delta W= & \int_{V}\{\delta u\}^{\mathrm{T}}\left\{\boldsymbol{F}_{V}\right\} \mathrm{d} V+\int_{A_{\mathrm{s} 1}}\{\delta u\}^{\mathrm{T}}\left\{\boldsymbol{F}_{A_{s}}\right\} \mathrm{d} A_{s} \\
& +\{\delta u\}^{\mathrm{T}}\left\{\boldsymbol{F}_{\mathrm{P}}\right\}-\int_{A_{\mathrm{s} 2}} \delta \phi \varsigma \mathrm{d} A_{s}-\delta \phi Q+\delta W_{\mathrm{D}}
\end{aligned}
$$

where $\left\{\boldsymbol{F}_{V}\right\}$ is the applied body force vector, $\left\{\boldsymbol{F}_{A_{\mathrm{s}}}\right\}$ is the applied surface force vector (defined on the surface $\left.A_{s 1}\right),\left\{\boldsymbol{F}_{\mathrm{P}}\right\}$ is the applied point load vector, $\phi$ is 
the electric potential, $\varsigma$ is the surface charge brought on surface $A_{s 2}, Q$ is the applied concentrated electric charge, and $\delta W_{\mathrm{D}}$ is the work done by damping forces. Figure 1 shows the various types of mechanical forces and electrical charges operating in the domain $V$. Substituting the non-linear electric enthalpy density function $H_{\text {non-lin }}$ and kinetic energy density $T_{\text {ke }}$ from equations (4) and (6) in equation (5), yields the following expression for the Hamilton's principle

$$
\begin{aligned}
- & \int_{t_{0}}^{t_{1}} \int_{V}\left[\rho\{\delta u\}^{\mathrm{T}}\{\ddot{u}\}\right]-\int_{t_{0}}^{t_{1}} \int_{V}\left[\{\delta \boldsymbol{S}\}^{\mathrm{T}}[\mathbf{C}]\{\boldsymbol{S}\}\right. \\
& -[\mathbf{C}]^{\mathrm{T}}[\mathbf{d}]^{\mathrm{T}}\{\boldsymbol{E}\}-[\operatorname{diag}(\{\boldsymbol{S}\})]\left[\boldsymbol{\gamma}_{11}\right]^{\mathrm{T}}\{\boldsymbol{E}\}-\frac{1}{2}\left[\gamma_{12}\right]^{\mathrm{T}}\left\{\mathbf{E}^{2}\right\} \\
& +[\operatorname{diag}(\{\boldsymbol{S}\})]\left[\mathbf{C}_{21}\right][\operatorname{diag}(\{\boldsymbol{S}\})]\{\boldsymbol{S}\}+\frac{1}{4}\left[\mathbf{C}_{22}\right]\left\{\boldsymbol{S}^{3}\right\} \\
& +\frac{3}{4}\left[\operatorname{diag}\left(\left\{\boldsymbol{S}^{2}\right\}\right)\right]\left[\mathbf{C}_{22}\right]^{\mathrm{T}}\{\boldsymbol{S}\}+\frac{1}{3}\left[\mathbf{C}_{1}\right]\left\{\boldsymbol{S}^{2}\right\} \\
& +\frac{2}{3}[\operatorname{diag}(\{\boldsymbol{S}\})]\left[\mathbf{C}_{1}\right]^{\mathrm{T}}\{\boldsymbol{S}\}-\left[\operatorname{diag}\left(\left\{\boldsymbol{S}^{2}\right\}\right)\right]\left[\boldsymbol{\gamma}_{21}\right]^{\mathrm{T}}\{\boldsymbol{E}\} \\
& \left.-[\operatorname{diag}(\{\boldsymbol{S}\})]\left[\boldsymbol{\gamma}_{22}\right]^{\mathrm{T}}\left\{\boldsymbol{E}^{2}\right\}-\frac{1}{3}\left[\boldsymbol{\gamma}_{23}\right]^{\mathrm{T}}\{\boldsymbol{E}\}^{3}\right] \mathrm{d} V \mathrm{~d} t \\
- & \int_{t_{0}}^{t_{1}} \int_{V}\{\delta E\}^{\mathrm{T}}\left[d[\mathbf{C}]\{\boldsymbol{S}\}+\left[\boldsymbol{v}_{0}\right]\{\boldsymbol{E}\}+\frac{1}{2}\left[\gamma_{11}\right]\left\{\boldsymbol{S}^{2}\right\}\right. \\
& +[\operatorname{diag}(\{\boldsymbol{E}\})]\left[\boldsymbol{\gamma}_{12}\right]\{\boldsymbol{S}\}+\frac{1}{3}\left[\boldsymbol{\gamma}_{13}\right]\left\{\boldsymbol{S}^{2}\right\} \\
& +\frac{2}{3}[\operatorname{diag}(\{\boldsymbol{E}\})]\left[\gamma_{13}\right]^{\mathrm{T}}\{\boldsymbol{E}\}+\frac{1}{3}\left[\boldsymbol{\gamma}_{21}\right]\left\{\boldsymbol{S}^{3}\right\} \\
& +[\operatorname{diag}(\{\boldsymbol{E}\})]\left[\gamma_{22}\right]\left\{\boldsymbol{S}^{2}\right\}+\left[\operatorname{diag}\left(\left\{\boldsymbol{E}^{2}\right\}\right)\right]\left[\boldsymbol{\gamma}_{23}\right]\{\boldsymbol{S}\} \\
& +\frac{1}{4}\left[\boldsymbol{v}_{1}\right]\left\{\boldsymbol{E}^{3}\right\}+\frac{3}{4}\left[\operatorname{diag}\left(\left\{\boldsymbol{E}^{2}\right\}\right)\right]\left[\boldsymbol{v}_{1}\right]^{\mathrm{T}}\{\boldsymbol{E}\} \\
& \left.+[\operatorname{diag}(\{\boldsymbol{E}\})]\left[\boldsymbol{v}_{2}\right][\operatorname{diag}(\{\boldsymbol{E}\})]\{\boldsymbol{E}\}\right] \mathrm{d} V \mathrm{~d} t \\
+ & \int_{t_{0}}^{t_{1}} \int_{V}\left[\{\delta u\}^{\mathrm{T}}\left\{\boldsymbol{F}_{v}\right\}\right] \mathrm{d} V \mathrm{~d} t+\int_{t_{0}}^{t_{1}} \int_{A_{s 1}}\left[\{\delta u\}^{\mathrm{T}}\left\{\mathbf{F}_{A_{s}}\right\}\right] \mathrm{d} A_{s} \mathrm{~d} t \\
+ & \int_{t_{0}}^{t_{1}}\{\delta u\}^{\mathrm{T}}\left\{\boldsymbol{F}_{\mathrm{P}}\right\} \mathrm{d} t-\int_{t_{0}}^{t_{1}} \int_{A_{s} 2} \delta \phi \zeta \mathrm{d} A_{s} \mathrm{~d} t \\
- & \int_{t_{0}}^{t_{1}} \delta \phi Q \mathrm{~d} t-\int_{t_{0}}^{t_{1}}\left[\delta W_{\mathrm{D}}\right] \mathrm{d} t=0
\end{aligned}
$$

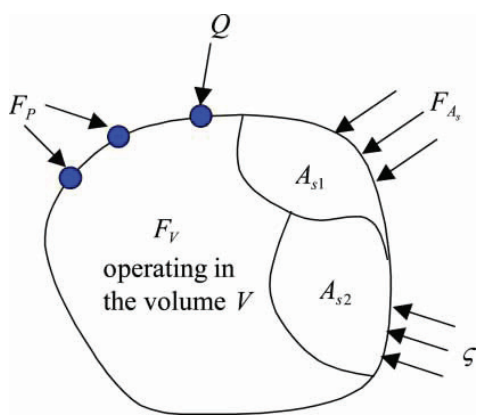

Fig. 1 Generalized mechanical and electrical forces of various types acting in the domain $V$ where the matrix with 'diag' refers to a diagonal matrix with the main diagonal terms being the terms of its vector. For deriving the expression for the virtual work done by damping forces, the proportional damping or Rayleigh damping model is considered and the formulation is given in next section.

\section{PROPORTIONAL DAMPING FORMULATION}

In this model, modal proportional damping is considered for the piezoceramic material and hence the virtual work done by viscous damping forces is given as (using the principle of Rayleigh proportional damping)

$$
\delta W_{\mathrm{D}}=\int_{V}\{\delta u\}^{\mathrm{T}}\left[\mathbf{C}_{\mathrm{damp}}\right]\{\dot{u}\} \mathrm{d} V
$$

where $\left[\mathbf{C}_{\mathrm{damp}}\right]$ is the proportional damping coefficient matrix and is expressed in terms of mass $[\mathbf{M}]$ and stiffness [ $\mathbf{K}]$ matrices with the help of constants $\alpha$ and $\beta$ as

$$
\left[\mathbf{C}_{\text {damp }}\right]=\alpha[\mathbf{M}]+\beta[\mathbf{K}]
$$

\section{THREE-DIMENSIONAL ANALYSIS OF A RECTANGULAR PIEZOCERAMIC PLATE}

The rectangular plate analysed in this work is shown in Fig. 2. One needs to perform three-dimensional analysis in order to determine all the elements of the non-linear material property matrices so that it can be used in the finite-element analysis to analyse other complex geometries made up of the same material. The form of solution for the non-linear displacement amplitude using Rayleigh-Ritz method and perturbation technique is described in the next section where three-dimensional matrices and vectors are used in the electric enthalpy function, kinetic energy, and virtual work.

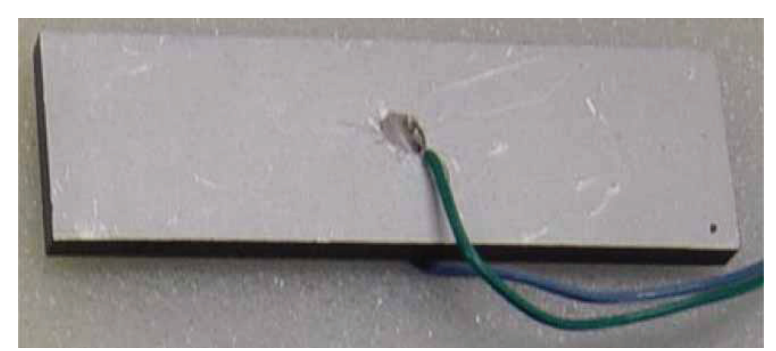

Fig. 2 Rectangular piezoceramic plate excited with electric field applied across its thickness 
Using the Hamilton's principle, one gets three partial differential equations for motions in three co-ordinate directions (i.e. $x, y$, and $z$ ). The displacement degrees of freedom used in the analysis are $u, v$, and $w$ along $x, y$, and $z$ directions. The governing equations are solved along with the boundary conditions to get the shape functions of the problem so that it can be used in Rayleigh-Ritz method to discretize the equations of motion and to solve by perturbation analysis. In this section, the solution of the problem will be discussed for deriving the shape functions for the three-dimensional problem corresponding to the first in-plane mode of free-free vibration of the rectangular plate. Again for simplification, it will be assumed that the electric field is constant in $z$-direction and the expression is given as follows

$$
E_{z}=-\frac{U_{0}}{h} \cos (\Omega t)
$$

In this three-dimensional analysis, all the stress and strain components exist in the formulation. The kinematic relations between strain and the displacement for the three-dimensional continuum are as follows

$$
\begin{aligned}
& S_{x x}=S_{1}=\frac{\partial u}{\partial x}, \quad S_{y y}=S_{2}=\frac{\partial v}{\partial y}, \quad S_{z z}=S_{3}=\frac{\partial w}{\partial z} \\
& S_{y z}=S_{4}=\frac{\partial v}{\partial z}+\frac{\partial w}{\partial y}, \quad S_{x z}=S_{5}=\frac{\partial u}{\partial z}+\frac{\partial w}{\partial x} \\
& S_{x y}=S_{6}=\frac{\partial u}{\partial y}+\frac{\partial v}{\partial x}
\end{aligned}
$$

The three-dimensional linear material property matrix [c] relating strains to stresses for three-dimensional continuum are given as

$$
\left\{\begin{array}{l}
T_{1} \\
T_{2} \\
T_{3} \\
T_{4} \\
T_{5} \\
T_{6}
\end{array}\right\}=\left[\begin{array}{cccccc}
c_{11} & c_{12} & c_{13} & 0 & 0 & 0 \\
c_{21} & c_{22} & c_{23} & 0 & 0 & 0 \\
c_{31} & c_{32} & c_{33} & 0 & 0 & 0 \\
0 & 0 & 0 & c_{44} & 0 & 0 \\
0 & 0 & 0 & 0 & c_{55} & 0 \\
0 & 0 & 0 & 0 & 0 & c_{66}
\end{array}\right]\left\{\begin{array}{l}
S_{1} \\
S_{2} \\
S_{3} \\
S_{4} \\
S_{5} \\
S_{6}
\end{array}\right\}
$$

\subsection{Derivation of the shape function of the three-dimensional linear eigenvalue problem}

The linear electric enthalpy function $H_{\text {linear }}$ corresponding to equation (2) has been used to derive the shape functions. After substituting the electric enthalpy density function in the Hamilton's principle, i.e. equation (5), and performing variation with respect to $u, v$, and $w$, the three equations of motion yielded, respectively, are

$$
\begin{aligned}
\rho \ddot{u}= & c_{11} \frac{\partial^{2} u}{\partial x^{2}}+c_{66} \frac{\partial^{2} u}{\partial y^{2}}+c_{55} \frac{\partial^{2} u}{\partial z^{2}}+\left(c_{12}+c_{66}\right) \frac{\partial^{2} v}{\partial x \partial y} \\
& +\left(c_{13}+c_{55}\right) \frac{\partial^{2} w}{\partial x \partial z} \\
\rho \ddot{v}= & \left(c_{21}+c_{66}\right) \frac{\partial^{2} u}{\partial x \partial y}+c_{66} \frac{\partial^{2} v}{\partial x^{2}}+c_{22} \frac{\partial^{2} v}{\partial y^{2}}+c_{44} \frac{\partial^{2} v}{\partial z^{2}} \\
& +\left(c_{23}+c_{44}\right) \frac{\partial^{2} w}{\partial y \partial z} \\
\rho \ddot{w}= & \left(c_{31}+c_{55}\right) \frac{\partial^{2} u}{\partial x \partial z}+\left(c_{32}+c_{44}\right) \frac{\partial^{2} v}{\partial y \partial z}+c_{55} \frac{\partial^{2} w}{\partial x^{2}} \\
& +c_{44} \frac{\partial^{2} w}{\partial y^{2}}+c_{33} \frac{\partial^{2} w}{\partial z^{2}}
\end{aligned}
$$

and the associated boundary conditions are given in Appendix 1. Using variable separable method, the solutions for $u, v$, and $w$ are as follows

$$
\begin{aligned}
u(x, y, z, t) & =U(x, y, z) p(t) ; v(x, y, z, t) \\
& =V(x, y, z) p(t) ; w(x, y, z, t) \\
& =W(x, y, z) p(t)
\end{aligned}
$$

Substituting $u(x, y, z, t), v(x, y, z, t)$, and $w(x, y, z, t)$ in equation (14) and separating the terms space and time, yields

$$
\begin{aligned}
& \frac{\ddot{p}(t)}{p(t)}=\text { constant }=-\omega^{2} \\
& c_{11} \frac{\partial^{2} U(x, y, z)}{\partial x^{2}}+c_{66} \frac{\partial^{2} U(x, y, z)}{\partial y^{2}}+c_{55} \frac{\partial^{2} U(x, y, z)}{\partial z^{2}} \\
& \quad+\left(c_{12}+c_{66}\right) \frac{\partial^{2} V(x, y, z)}{\partial x \partial y}+\left(c_{13}+c_{55}\right) \frac{\partial^{2} W(x, y, z)}{\partial x \partial z} \\
& \quad=-\rho \omega^{2} U(x, y, z) \\
& \left(c_{21}+c_{66}\right) \frac{\partial^{2} U(x, y, z)}{\partial x \partial y}+c_{66} \frac{\partial^{2} V(x, y, z)}{\partial x^{2}} \\
& \quad+c_{22} \frac{\partial^{2} V(x, y, z)}{\partial y^{2}}+c_{44} \frac{\partial^{2} V(x, y, z)}{\partial z^{2}} \\
& \quad+\left(c_{23}+c_{44}\right) \frac{\partial^{2} W(x, y, z)}{\partial y \partial z}=-\rho \omega^{2} V(x, y, z) \\
& \left(c_{31}+c_{55}\right) \frac{\partial^{2} U(x, y, z)}{\partial x \partial z}+\left(c_{32}+c_{44}\right) \frac{\partial^{2} V(x, y, z)}{\partial y \partial z} \\
& \quad+c_{55} \frac{\partial^{2} W(x, y, z)}{\partial x^{2}}+c_{44} \frac{\partial^{2} W(x, y, z)}{\partial y^{2}} \\
& \quad+c_{33} \frac{\partial^{2} W(x, y, z)}{\partial z^{2}}=-\rho \omega^{2} W(x, y, z)
\end{aligned}
$$


The solutions can be assumed as

$$
\begin{aligned}
& U(x, y, z)=u_{0} \sin \left(\lambda_{1} x\right) \cos \left(\lambda_{2} y\right) \cos \left(\lambda_{3} y\right) \\
& V(x, y, z)=v_{0} \cos \left(\lambda_{1} x\right) \sin \left(\lambda_{2} y\right) \cos \left(\lambda_{3} y\right) \\
& W(x, y, z)=w_{0} \cos \left(\lambda_{1} x\right) \cos \left(\lambda_{2} y\right) \sin \left(\lambda_{3} y\right) \\
& p(t)=\sin (\omega t) \text { or } \cos (\omega t)
\end{aligned}
$$

(depending upon the initial condition)

Substituting the assumed solutions into the equation (14), yields the eigenvalue equation as

$$
\begin{aligned}
& {\left[\begin{array}{ccc}
c_{11} \lambda_{1}^{2}+c_{66} \lambda_{2}^{2} & \left(c_{12}+c_{66}\right) \lambda_{1} \lambda_{2} & \left(c_{13}+c_{55}\right) \lambda_{1} \lambda_{3} \\
+c_{55} \lambda_{3}^{2} & & \\
\left(c_{21}+c_{66}\right) \lambda_{1} \lambda_{2} & c_{66} \lambda_{1}^{2}+c_{22} \lambda_{2}^{2} & \left(c_{23}+c_{44}\right) \lambda_{2} \lambda_{3} \\
\left(c_{31}+c_{55}\right) \lambda_{1} \lambda_{3} & \left(c_{34}+c_{44}\right) \lambda_{2} \lambda_{3} & c_{55} \lambda_{1}^{2}+c_{44} \lambda_{2}^{2} \\
+c_{33} \lambda_{3}^{2}
\end{array}\right]} \\
& \quad \times\left\{\begin{array}{c}
u_{0} \\
v_{0} \\
w_{0}
\end{array}\right\}=\rho \omega^{2}\left\{\begin{array}{c}
u_{0} \\
v_{0} \\
w_{0}
\end{array}\right\}
\end{aligned}
$$

Solving the eigenvalue equations (i.e. putting the determinant of the matrix to be zero), yields the relationship between the wave numbers (i.e. $\lambda_{1}, \lambda_{2}$, and $\lambda_{3}$ ) and the eigenfrequencies $\omega$. The assumed solutions must satisfy all the boundary conditions given in Appendix 1 (for short-circuited electrodes, i.e. $E_{z}=0$ ). Substituting the expressions for $u$, $w$, and $v$ in the boundary conditions yields various combinations of the five unknowns (i.e. $v_{0} / u_{0}, w_{0} / u_{0}$, $\lambda_{1}, \lambda_{2}$, and $\lambda_{3}$ ). Hence, finally $u, v$, and $w$ become summation of various combinations of the terms containing $\left(\lambda_{1}\right)_{i},\left(\lambda_{2}\right)_{j}$, and $\left(\lambda_{3}\right)_{k}$, where $i, j$, and $k$ refer to each possible solution. Hence $u(x, y, z, t), v(x, y, z, t)$, and $w(x, y, z, t)$ can be written as

$$
\begin{aligned}
u(x, y, z, t)= & \sum_{i=1, n} \sum_{j=1, n} \sum_{k=1, n} u_{0 i j k} \sin \left(\lambda_{1}\right)_{i} \\
& \times x \cos \left(\lambda_{2}\right)_{j} y \cos \left(\lambda_{3}\right)_{k} z p_{i j k}(t) \\
v(x, y, z, t)= & \sum_{i=1, n} \sum_{j=1, n} \sum_{k=1, n} v_{0 i j k} \cos \left(\lambda_{1}\right)_{i} \\
& \times x \sin \left(\lambda_{2}\right)_{j} y \cos \left(\lambda_{3}\right)_{k} z p_{i j k}(t) \\
w(x, y, z, t)= & \sum_{i=1, n} \sum_{j=1, n} \sum_{k=1, n} w_{0 i j k} \cos \left(\lambda_{1}\right)_{i} \\
& \times x \cos \left(\lambda_{2}\right)_{j} y \sin \left(\lambda_{3}\right)_{k} z p_{i j k}(t)
\end{aligned}
$$

For the first longitudinal mode of the plate (when length $(l)>$ width $(b)>$ height $(h)$ ), the solutions for

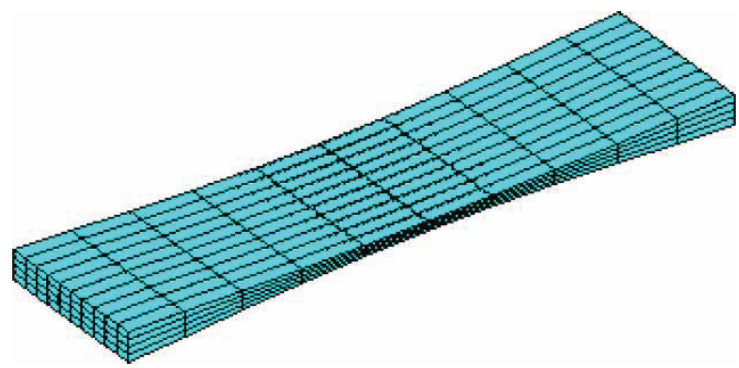

Fig. 3 Mode-shape of the plate in the first free-free in-plane longitudinal vibration mode

$U(x, y, z), V(x, y, z)$, and $W(x, y, z)$ are given as

$$
\begin{aligned}
& \lambda_{1}=\lambda_{2}=\lambda_{3}=\frac{\pi}{l} ; \quad \frac{v_{0}}{u_{0}}=-\frac{c_{21} c_{33}-c_{31} c_{23}}{c_{22} c_{33}-c_{32} c_{23}} \\
& \frac{w_{0}}{u_{0}}=-\frac{c_{21} c_{32}-c_{31} c_{22}}{c_{22} c_{32}-c_{22} c_{33}} \\
& U(x, y, z)=u_{0} \sin \left(\frac{\pi x}{l}\right) \cos \left(\frac{\pi y}{l}\right) \cos \left(\frac{\pi z}{l}\right) \\
& V(x, y)=v_{0} \cos \left(\frac{\pi x}{l}\right) \sin \left(\frac{\pi y}{l}\right) \cos \left(\frac{\pi z}{l}\right) \\
& W(x, y)=w_{0} \cos \left(\frac{\pi x}{l}\right) \cos \left(\frac{\pi y}{l}\right) \sin \left(\frac{\pi z}{l}\right)
\end{aligned}
$$

Using these shape functions, the non-linear equations of motion for the maximum amplitude $A$ using the procedure can be solved as described in the next section. The mode shape of the plate for the first free-free inplane mode is shown in Fig. 3 and the three shape functions $U(x, y, z), V(x, y, z)$, and $W(x, y, z)$ for the same mode are shown in Figs 4(a) to (c), respectively.

\subsection{Solution for the non-linear analysis with proportional damping formulation}

The Rayleigh-Ritz method has been used to solve the non-linear equations represented by the variational equation (8) and the displacement functions used in the analysis represented by equation (15). These functions are substituted into the variational equation form of the Hamilton's principle containing non-linear enthalpy function $H_{\text {non-lin }}$ from equation (4). The shape functions corresponding to the mode $k$ nearest to the excitation frequency alone are used. For the first longitudinal vibration mode of the plate, the mode shape functions as given by equation (20) are used in this analysis. After performing the variation of the expression in the Hamilton's principle with respect to $p_{k}(t)$, yields the discretized non-linear equation of motion as

$$
\begin{aligned}
m_{k} \ddot{p}_{k}(t)+c_{k} p_{k}(t)+B_{k} p_{k}^{2}(t)+\varepsilon_{k} p_{k}^{3}(t) \\
=f_{k}^{(1)} \cos \Omega t+f_{k}^{(2)} p_{k}(t) \cos \Omega t+f_{k}^{(3)} p_{k}^{2}(t) \cos \Omega t \\
\quad+f_{k}^{(4)} \cos ^{2} \Omega t+f_{k}^{(5)} p_{k}(t) \cos ^{2} \Omega t+f_{k}^{(6)} \cos ^{3} \Omega t
\end{aligned}
$$



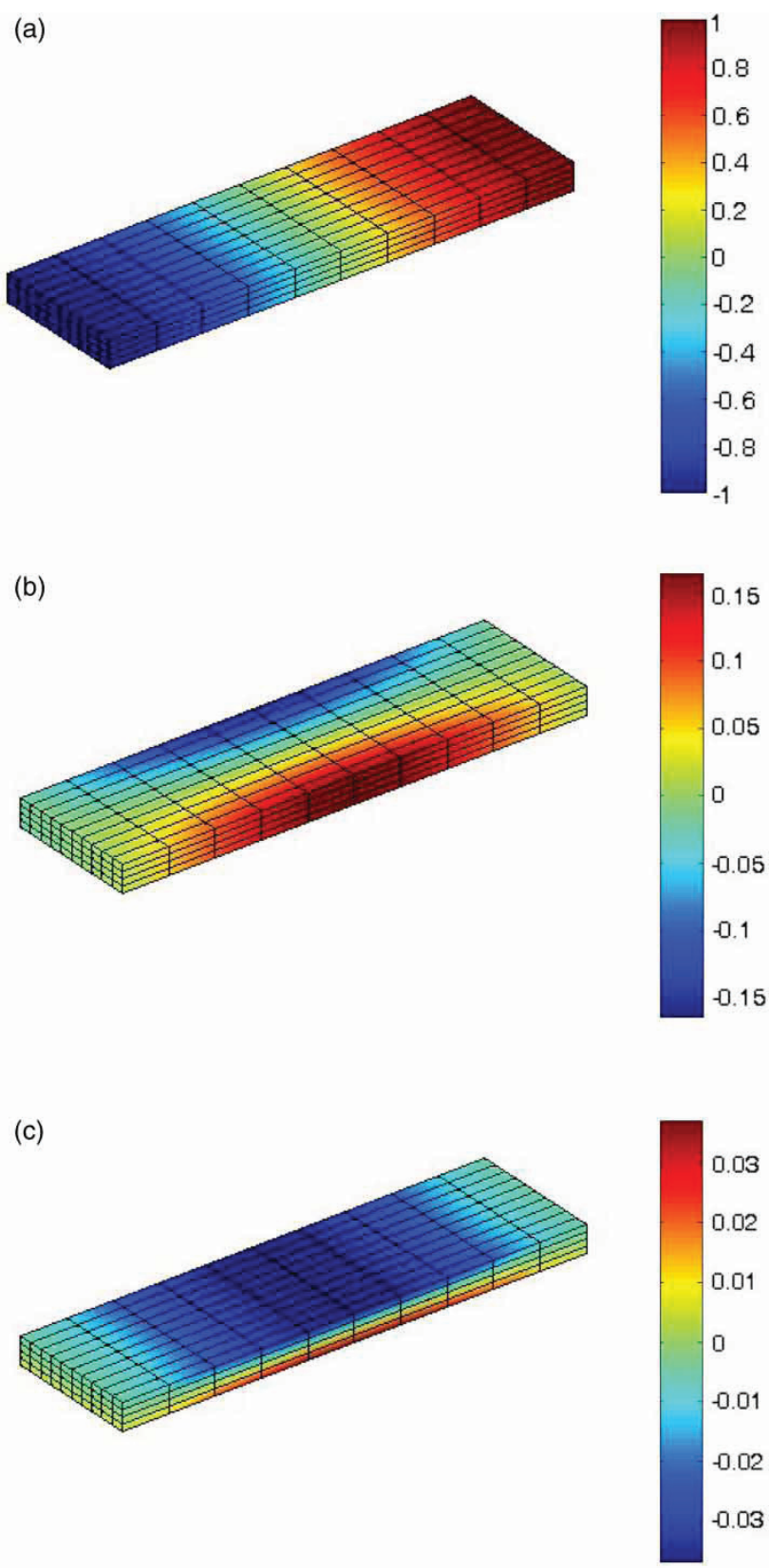

Fig. 4 Contour plot of three-dimensional shape functions for the first in-plane free-free longitudinal vibration mode of the plate on the original plate geometry: (a) $U(x, y, z)$; (b) $V(x, y, z)$; (c) $W(x, y, z)$

where $k=1,2,3, \ldots$ and the coefficients are given in Appendix 2.

\subsection{Solution by perturbation analysis for the formulation with proportional damping}

An approximate solution for the non-linear equation, i.e. equation (21), can be found using perturbation technique [29]. Linear modal proportional damping with the non-dimensional damping coefficient $\xi$ has been used in this analysis. One can nondimensionalize time as

$$
\tau=\omega_{0} t
$$

where $\omega_{0}=\sqrt{c_{k} / m_{k}}$ is the natural frequency of the $k$ th mode of the system. Equation (21) can now be written as

$$
\begin{aligned}
p^{\prime \prime}+ & \varepsilon D^{*} p^{\prime}+p+\varepsilon \chi p^{2}+\varepsilon \alpha^{*} p^{3} \\
= & \varepsilon\left(h_{1} \cos \eta \tau+h_{2} \sin \eta \tau\right)\left(1+\psi_{0} p+\beta^{*} p^{2}\right) \\
& +\varepsilon^{2} \gamma\left(h_{1} \cos \eta \tau+h_{2} \sin \eta \tau\right)^{2}\left(1+\psi_{1} p\right) \\
& +\varepsilon^{3} \delta^{*}\left(h_{1} \cos \eta \tau+h_{2} \sin \eta \tau\right)^{3}
\end{aligned}
$$

where

$$
\begin{aligned}
& ()^{\prime}=\frac{\partial}{\partial \tau}, \quad \eta=\frac{\Omega}{\omega_{0}}, \quad D^{*}=\frac{2 \xi}{\varepsilon}, \quad \chi=\frac{B_{k}}{m_{k} \omega_{0}^{2} \varepsilon} \\
& h_{1}^{2}+h_{2}^{2}=h_{0}^{2}, \quad h_{0}=\frac{f_{k}^{(1)}}{m_{k} \omega_{0}^{2} \varepsilon}, \quad \psi_{0}=\frac{f_{k}^{(2)}}{f_{k}^{(1)}} \\
& \beta^{*}=\frac{f_{k}^{(3)}}{f_{k}^{(1)}}, \quad \gamma=\frac{f_{k}^{(4)}}{m_{k} \omega_{0}^{2} \varepsilon^{2} h_{0}^{2}}, \quad \psi_{1}=\frac{f_{k}^{(5)}}{f_{k}^{(4)}} \\
& \delta^{*}=\frac{f_{k}^{(6)}}{m_{k} \omega_{0}^{2} \varepsilon^{3} h_{0}^{3}}
\end{aligned}
$$

The coefficients $h_{1}$ and $h_{2}$ are introduced in order to allow for a phase shift between the excitation and the response of the system due to the presence of damping. The parameter $\varepsilon$ is called the perturbation parameter and can be chosen arbitrarily. But, it should be very small, e.g. 0.001. Now, the Lindstedt-Poincaré method is used with the following expansion for $p$ and $\eta$.

$$
p=p_{0}+\varepsilon p_{1}+\cdots, \quad \eta=\eta_{0}+\varepsilon \eta_{1}+\cdots
$$

Substituting equation (25) in equation (23) and collecting the coefficients for $\varepsilon^{0}$ and $\varepsilon^{1}$, yields

$$
\begin{aligned}
p_{0}^{\prime \prime}+\eta^{2} p_{0}= & 0 \\
p_{1}^{\prime \prime}+\eta^{2} p_{1}= & -D^{*} p_{0}^{\prime}+2 \eta_{1} p_{0}-\chi p_{0}^{2}-\alpha^{*} p_{0}^{3} \\
& +\left(h_{1} \cos \eta \tau+h_{2} \sin \eta \tau\right) \\
& \times\left(1+\psi_{0} p_{0}+\beta^{*} p_{0}^{2}\right)
\end{aligned}
$$

The above expressions were obtained by using the following expansion

$$
\begin{gathered}
1=\eta-\varepsilon \eta_{1}(\text { taking only the first two terms } \\
\quad \text { of the expansion of } \eta) \\
\Rightarrow 1^{2}=\left(\eta-\varepsilon \eta_{1}\right)^{2}
\end{gathered}
$$


The solution of first part of equation (26) can be written as

$$
p_{0}=A \cos \eta \tau
$$

where the amplitude $A$ is unknown. By substituting equation (28) for $p_{0}$ in second part of equation (26), one gets

$$
\begin{aligned}
p_{1}^{\prime \prime}+\eta^{2} p_{1}= & \xi(\eta A \sin \eta \tau)+2 \eta_{1} A \cos \eta \tau \\
& -\chi A^{2} \cos ^{2} \eta \tau-\alpha^{*} A^{3} \cos ^{3} \eta \tau \\
& +h_{1} \cos \eta \tau+h_{1} \psi_{0} A \cos ^{2} \eta \tau \\
& +h_{1} \beta^{*} A^{2} \cos ^{3} \eta \tau+h_{2} \sin \eta \tau \\
& +h_{2} \psi_{0} A \sin \eta \tau \cos \eta \tau \\
& +h_{2} \beta^{*} A^{2} \sin \eta \tau \cos ^{2} \eta \tau
\end{aligned}
$$

Rewriting equation (29) in terms of $\sin \eta \tau, \cos \eta \tau$, $\sin 2 \eta \tau, \cos 2 \eta \tau, \sin 3 \eta \tau$, and $\cos 3 \eta \tau$, one gets

$$
\begin{aligned}
p_{1}^{\prime \prime}+ & \eta^{2} p_{1} \\
= & \left(\xi \eta A+h_{2}+\frac{1}{4} h_{2} \beta^{*} A^{2}\right) \sin \eta \tau \\
& +\left(2 \eta_{1} A-\frac{3}{4} \alpha^{*} A^{3}+h_{1}+\frac{3}{4} h_{1} \beta^{*} A^{2}\right) \cos \eta \tau \\
& +\left(-\frac{1}{2} \xi A^{2}+\frac{1}{2} h_{1} \psi_{0} A\right)+\frac{1}{2} h_{2} \psi_{0} A \sin 2 \eta \tau \\
& +\frac{1}{2}\left(h_{1} \psi_{0} A-\xi A^{2}\right) \cos 2 \eta \tau+\frac{1}{4} h_{2} \beta^{*} A^{2} \sin 3 \eta \tau \\
& +\frac{1}{4}\left(h_{1} \beta^{*} A^{2}-\alpha^{*} A^{3}\right) \cos 3 \eta \tau
\end{aligned}
$$

For the solution of equation (30) to remain finite, the secular terms (terms with $\sin \eta \tau$ and $\cos \eta \tau$ ) must be zero [29]. Hence, one gets

$$
h_{1}=\frac{-2 \eta_{1} A+(3 / 4) \alpha^{*} A^{3}}{1+(3 / 4) \beta^{*} A^{2}}, \quad h_{2}=\frac{-D^{*} A \eta}{1+(1 / 4) \beta^{*} A^{2}}
$$

Combining $h_{1}$ and $h_{2}$, one gets $h_{0}$ which is known and this relation reduces to a polynomial equation in the unknown stationary amplitude $A$ which is expressed as

$$
a_{5} A^{10}+a_{4} A^{8}+a_{3} A^{6}+a_{2} A^{4}+a_{1} A^{2}+a_{0}=0
$$

where the coefficients are written as

$$
\begin{aligned}
& a_{5}=\frac{9}{256} \alpha^{* 2} \beta^{* 2} \\
& a_{4}=\frac{9}{32} \alpha^{* 2} \beta^{*}-\frac{9}{256} \beta^{* 4} h_{0}^{2}-\frac{3}{16} \beta^{* 2} \eta_{1} \alpha^{*}
\end{aligned}
$$

$$
\begin{aligned}
a_{3}= & \frac{9}{16} \alpha^{* 2}-\frac{3}{8} \beta^{* 3} h_{0}^{2} \\
& +\frac{9}{16} \beta^{* 2} D^{* 2} \eta^{2}-\frac{3}{2} \alpha^{*} \beta^{*} \eta_{1}+\frac{1}{4} \beta^{* 2} \eta_{1}^{2} \\
a_{2}= & -\frac{11}{8} \beta^{* 2} h_{0}^{2}+\frac{3}{2} \beta^{*} D^{* 2} \eta^{2}-3 \alpha^{*} \eta_{1}+2 \beta^{*} \eta_{1}^{2} \\
a_{1}= & -2 \beta^{*} h_{0}^{2}+D^{* 2} \eta^{2}+4 \eta_{1}^{2}, \quad a_{0}=-h^{2}
\end{aligned}
$$

The roots of the polynomial represent the solutions for the stationary amplitude $A$. In the region of the response curve where jump phenomenon is observed, it will give three real roots and two imaginary roots. The three real roots correspond to sweep-up frequency response, sweep-down frequency response, and the unstable responses, respectively. At other regions, the polynomial will have only one real root and four imaginary roots and hence there is a unique response for both sweep-up and sweep-down analyses. The total response corresponding to the series expansion of $p$ is given by (neglecting higher-order terms like $\sin 2 \eta \tau$, $\cos 2 \eta \tau, \sin 3 \eta \tau$, and $\cos 3 \eta \tau)$

$$
p=p_{0}+\varepsilon p_{1}=A \cos \eta \tau+\frac{\varepsilon}{2 \eta^{2}}\left(h_{1} \psi_{0} A-\chi A^{2}\right)
$$

After the amplitude of displacement is calculated, current flowing through the piezoceramic can be calculated following the method for current calculation as described in next section.

\subsection{Calculation of current flowing through the piezoceramic plate}

The current flowing through the piezoelectric plate can be calculated using the expression for the electric displacement as follows

$$
I(t)=\int_{A_{\mathrm{s}}} \dot{D}_{z}(t) \mathrm{d} A_{\mathrm{s}}
$$

where $A_{\mathrm{s}}$ is the area through which current is flowing in the piezoceramic plate and $z$ is the direction perpendicular to the area $A_{\mathrm{s}}$ and $\dot{D}_{z}$ is the time derivative of the $z$-component of the electric displacement vector $\{\boldsymbol{D}\}$. The electric displacement vector $\{\boldsymbol{D}\}$ can be calculated from the generalized non-linear electric enthalpy density function as

$$
\{\boldsymbol{D}\}=-\frac{\partial H_{\text {non-lin }}}{\partial\{\boldsymbol{E}\}}
$$

Using equation (4) for $H_{\text {non-lin }}$ in the equation (36) and taking time derivative, one can write $\{\dot{\boldsymbol{D}}\}$ as 


$$
\begin{aligned}
\{\dot{D}\}= & {[\mathbf{d}][\mathbf{c}]\{\dot{S}\}+\left[\boldsymbol{v}_{0}\right]\{\dot{E}\}+\left[\boldsymbol{\gamma}_{11}\right]\{S \dot{S}\} } \\
& +[\operatorname{diag}(\{\dot{E}\})]\left[\boldsymbol{\gamma}_{12}\right]\{\boldsymbol{S}\}+[\operatorname{diag}(\{\boldsymbol{E}\})]\left[\gamma_{12}\right]\{\dot{S}\} \\
& +\frac{2}{3}\left[\gamma_{13}\right]\{E \dot{E}\}+\frac{2}{3}[\operatorname{diag}(\{\dot{E}\})]\left[\boldsymbol{\gamma}_{13}\right]^{\mathrm{T}}\{E\} \\
& +\frac{2}{3}[\operatorname{diag}(\{\boldsymbol{E}\})]\left[\boldsymbol{\gamma}_{13}\right]^{\mathrm{T}}\{\dot{E}\}+\left[\boldsymbol{\gamma}_{21}\right]\left\{S^{2} \dot{S}\right\} \\
& +[\operatorname{diag}(\{\dot{E}\})]\left[\boldsymbol{\gamma}_{22}\right]\left\{S^{2}\right\}+2[\operatorname{diag}(\{\boldsymbol{E}\})]\left[\boldsymbol{\gamma}_{22}\right] \\
& \times\{S \dot{S}\}+2[\operatorname{diag}(\{E \dot{E}\})]\left[\boldsymbol{\gamma}_{23}\right]\{S\} \\
& +\left[\operatorname{diag}\left(\left\{\boldsymbol{E}^{2}\right\}\right)\right]\left[\boldsymbol{\gamma}_{23}\right]\{\dot{S}\}+\frac{3}{4}\left[\boldsymbol{v}_{1}\right]\left\{\boldsymbol{E}^{2} \dot{E}\right\} \\
& +\frac{3}{2}[\operatorname{diag}(\{E \dot{E}\})]\left[\boldsymbol{v}_{1}\right]\{\boldsymbol{E}\}+\frac{3}{4}\left[\operatorname{diag}\left(\left\{\boldsymbol{E}^{2}\right\}\right)\right] \\
& \times\left[\boldsymbol{v}_{1}\right]^{\mathrm{T}}\{\dot{E}\}+\frac{1}{2}[\operatorname{diag}(\{\dot{E}\})]\left[\boldsymbol{v}_{2}\right][\operatorname{diag}(\{\boldsymbol{E}\})] \\
& \times\{\boldsymbol{E}\}+[\operatorname{diag}(\{\boldsymbol{E}\})]\left[\boldsymbol{v}_{2}\right][\operatorname{diag}(\{\boldsymbol{E}\})]\{\dot{E}\} \\
& +\frac{1}{2}[\operatorname{diag}(\{\dot{E}\})]\left[\boldsymbol{v}_{2}\right]^{\mathrm{T}}[\operatorname{diag}(\{\boldsymbol{E}\})]\{\boldsymbol{E}\} \\
& +[\operatorname{diag}(\{\boldsymbol{E}\})]\left[\boldsymbol{v}_{2}\right]^{\mathrm{T}}[\operatorname{diag}(\{\boldsymbol{E}\})]\{\dot{E}\}
\end{aligned}
$$

\section{RESULTS AND DISCUSSION}

\subsection{Prediction of non-linear response of the PIC 181 rectangular plate in the first in-plane mode}

Experiments have been conducted on a rectangular piezoelectric plate made of PIC 181 piezoceramic manufactured by PI Ceramic, Lederhose, Germany.

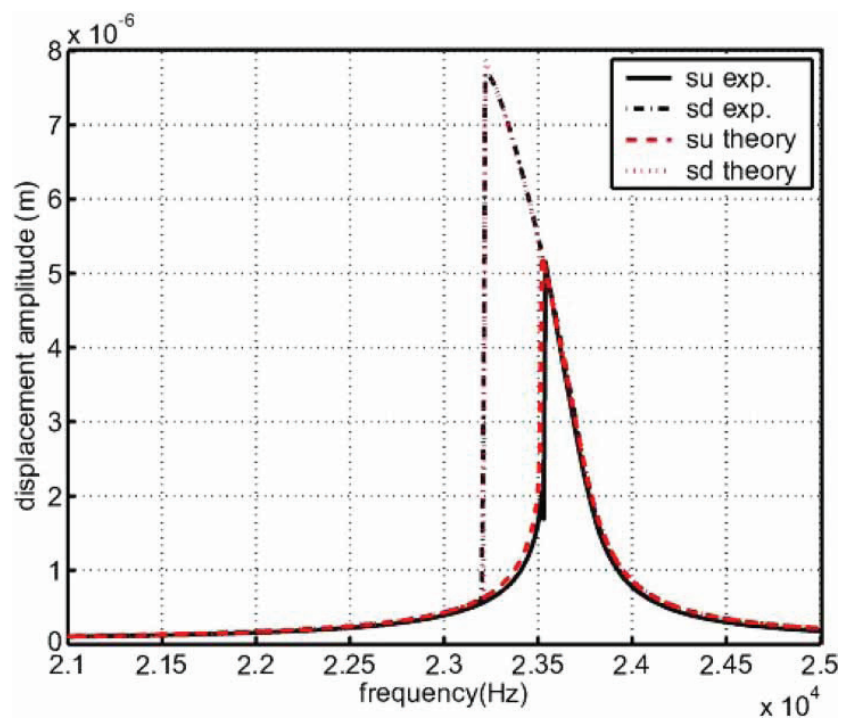

Fig. 5 Displacement response of the PIC 181 plate for the applied sinusoidal electric field of $7.5 \mathrm{~V} / \mathrm{mm}$ peak amplitude (first in-plane mode)
Figure 2 shows the plate with the wires attached on two faces of the plate coated with electrode material. The electric field is applied along the thickness, and the vibration amplitude of the plate is measured at the end of its length in the in-plane free-free mode by using a laser vibrometer (velocity amplitude from the laser vibrometer is converted to the displacement amplitude by dividing it with the circular frequency $\omega$ of the corresponding mode of excitation of the plate). The current flowing through the piezoelectric plate is measured by connecting a resistance in series with the plate and measuring the potential drop across the resistance (the potential difference is converted to the current by dividing it with the known value

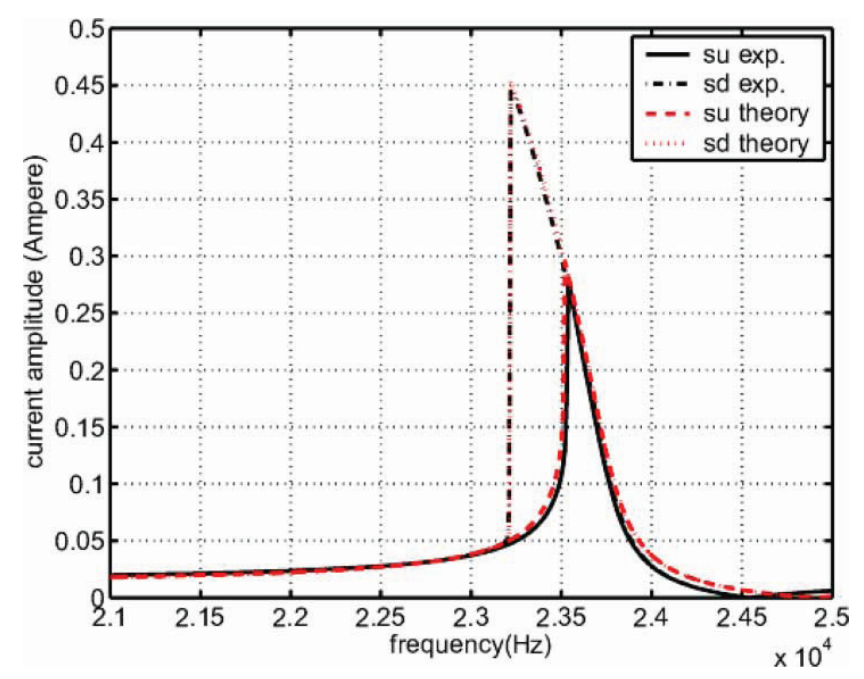

Fig. 6 Current response of the PIC 181 plate for the applied sinusoidal electric field of $7.5 \mathrm{~V} / \mathrm{mm}$ peak amplitude (first in-plane mode)

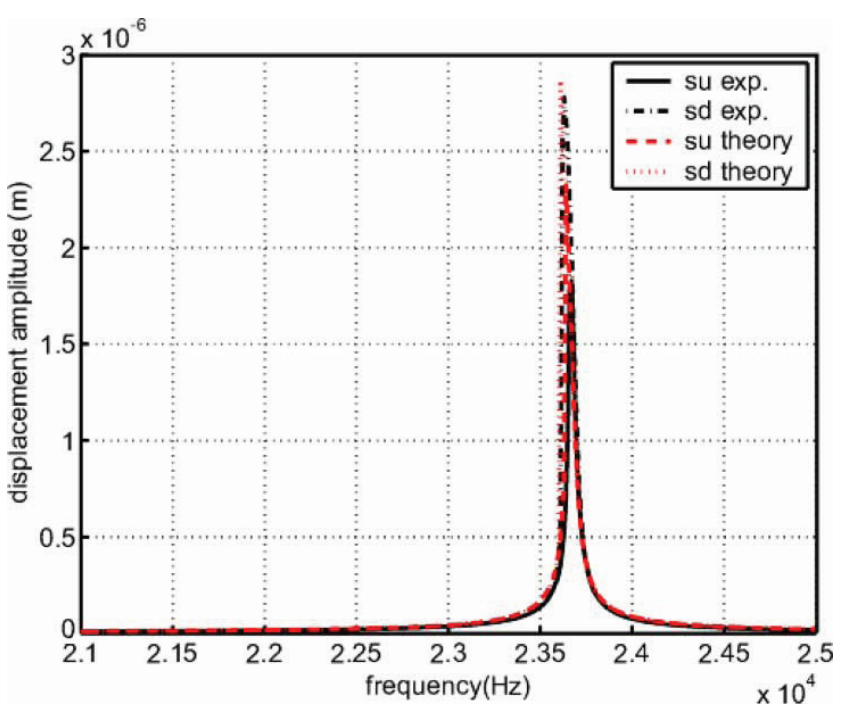

Fig. 7 Displacement response of the PIC 181 plate for the applied sinusoidal electric field of $0.75 \mathrm{~V} / \mathrm{mm}$ peak amplitude (first in-plane mode) 
of resistance of the resistor). The material properties used in the analysis are given in Appendix 3 (Fig. 25 for the damping coefficient). These material properties have been obtained by optimization of the above closed form solution with experimental results and the details are given elsewhere $[\mathbf{3 0}, \mathbf{3 1}])$. The length, width, and thickness of the plate are 70,20 , and $4 \mathrm{~mm}$, respectively.

In order to study the non-linear response of the above-said piezoceramic plate, it was excited with applied sinusoidal electric fields of different amplitudes. One of the non-linear phenomena observed is the jump behaviour, which appears when the plate is excited with different frequencies near the resonance region (e.g. the resonance of the linear system is at $23.65 \mathrm{kHz}$ for the first in-plane longitudinal mode). When the frequency of excitation is gradually increased from 21 to $25 \mathrm{kHz}$, the amplitude of displacement increases gradually (Fig. 5) and then the amplitude gets increased suddenly (jump for the

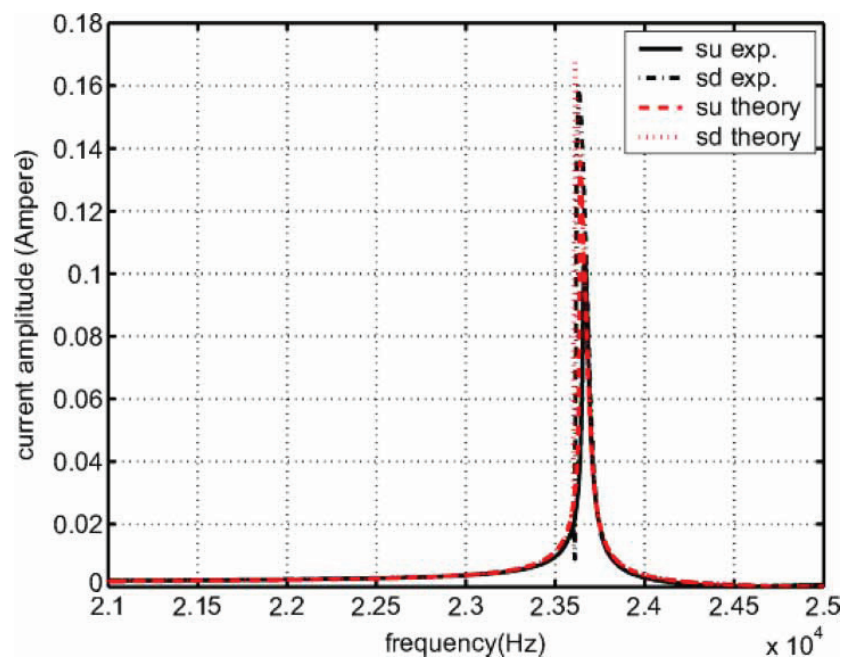

Fig. 8 Current response of the PIC 181 plate for the applied sinusoidal electric field of $0.75 \mathrm{~V} / \mathrm{mm}$ peak amplitude (first in-plane mode)

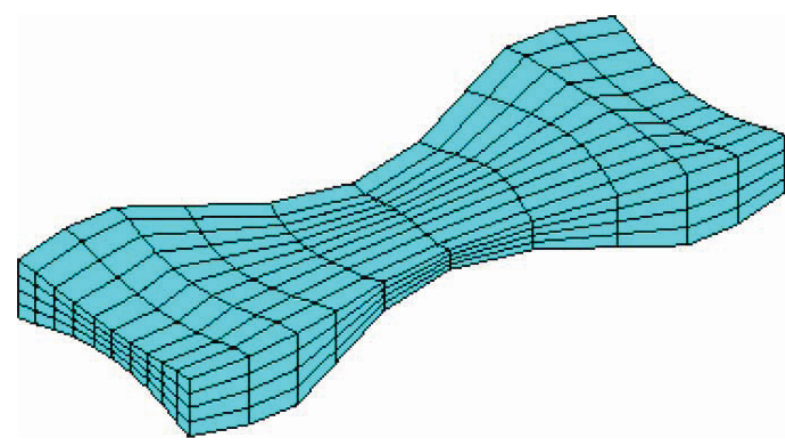

Fig. 9 Mode-shape of the piezoceramic plate for the second free-free in-plane longitudinal vibration mode sweep-up mode) at a frequency that depends upon the excitation field (e.g. jump is at $23.51 \mathrm{kHz}$ for electric field amplitude of $7.5 \mathrm{~V} / \mathrm{mm}$ ). Similarly, when the frequency of excitation is decreased gradually from 25 to $21 \mathrm{kHz}$, the amplitude of displacement increases gradually (Fig. 5) and then the amplitude gets decreased suddenly (jump for the sweep-down mode) at a frequency that depends again upon the excitation field (e.g. jump is at $23.23 \mathrm{kHz}$ for electric field amplitude of $7.5 \mathrm{~V} / \mathrm{mm}$ ). The above jump phenomenon reflects
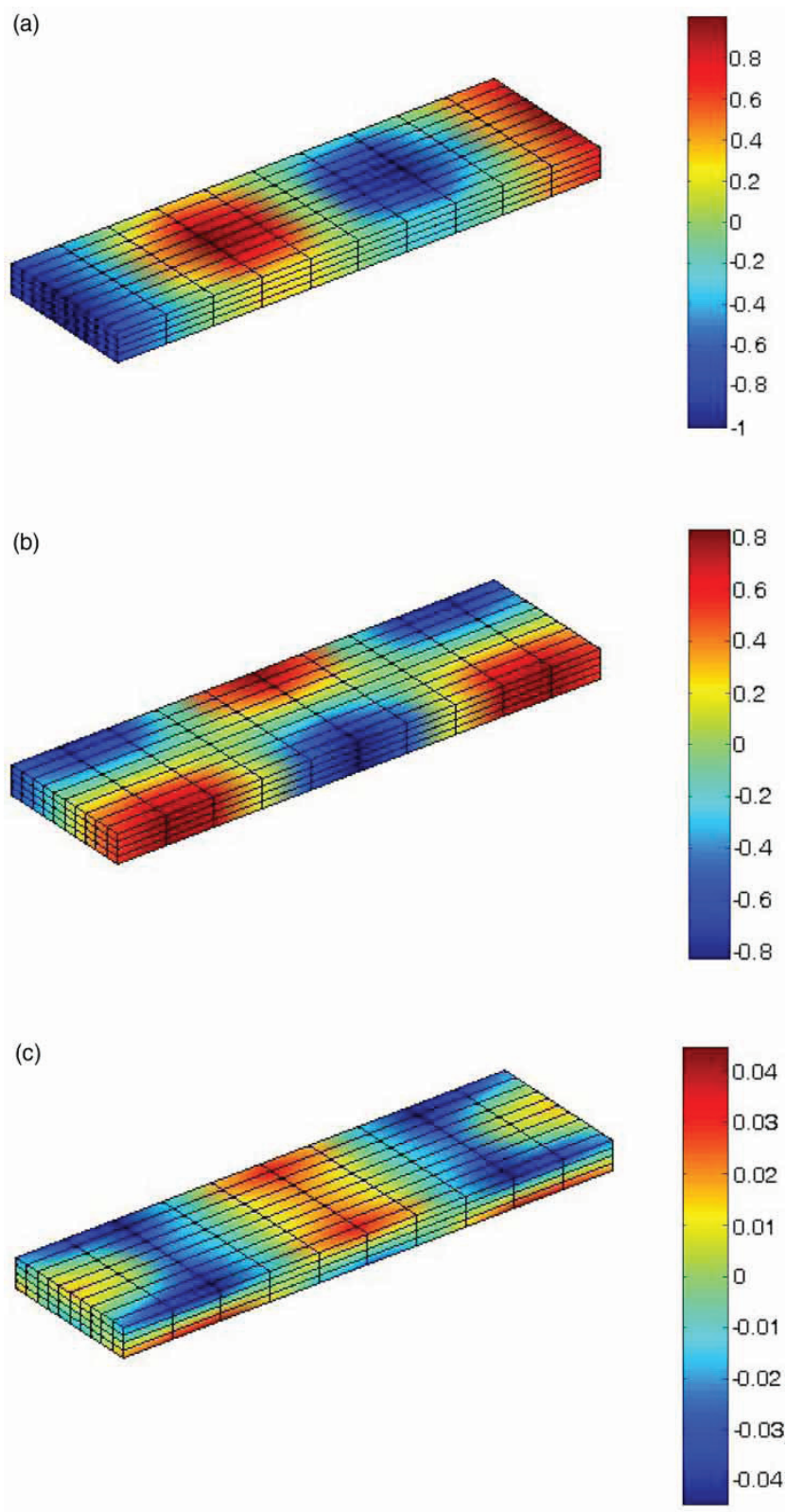

Fig. 10 Contour plot of three-dimensional shape functions for the second in-plane free-free longitudinal vibration mode of the plate on the original plate geometry: (a) $U(x, y, z)$; (b) $V(x, y, z)$; (c) $W(x, y, z)$ 
that the piezoelectric plate behaves like a softening spring (with cubic non-linear behaviour [29] where the spring stiffness changes with applied frequency of excitation), where the response depends upon the path (i.e. increasing or decreasing frequency amplitude) of excitation. In order to capture this effect, one must include cubic terms in the material constitutive equation for the electrical, mechanical, and coupled piezoelectric domains and the hence the same are included in the generalized three-dimensional electric enthalpy function of equation (4).

The results of analysis with a high electric field (viz. $7.5 \mathrm{~V} / \mathrm{mm}$ ) and with a low electric field (viz. $0.75 \mathrm{~V} / \mathrm{mm}$ )

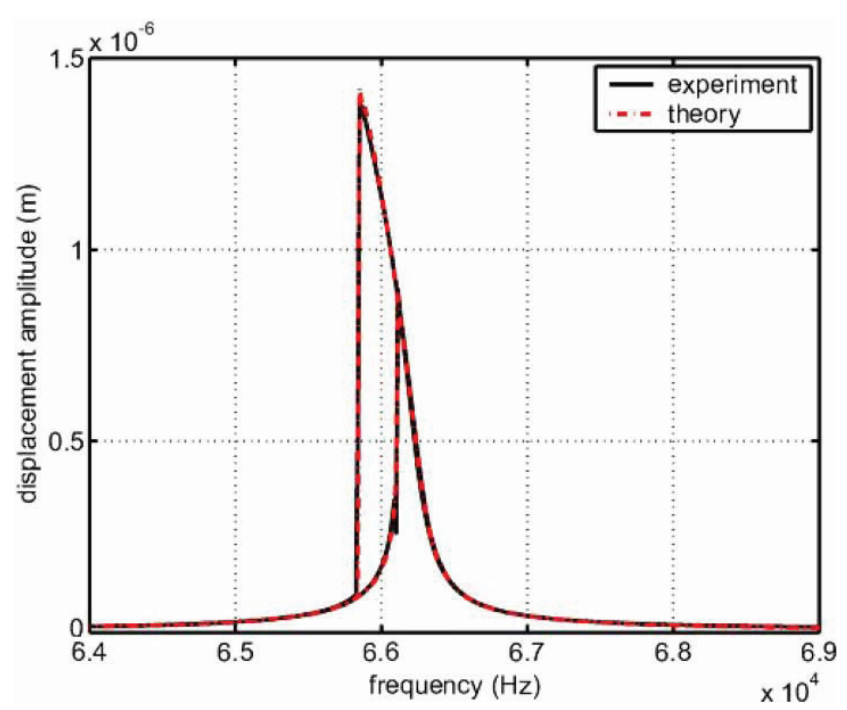

Fig. 11 Displacement response of the PIC 181 plate for the applied sinusoidal electric field of $7.5 \mathrm{~V} / \mathrm{mm}$ peak amplitude (second in-plane mode)

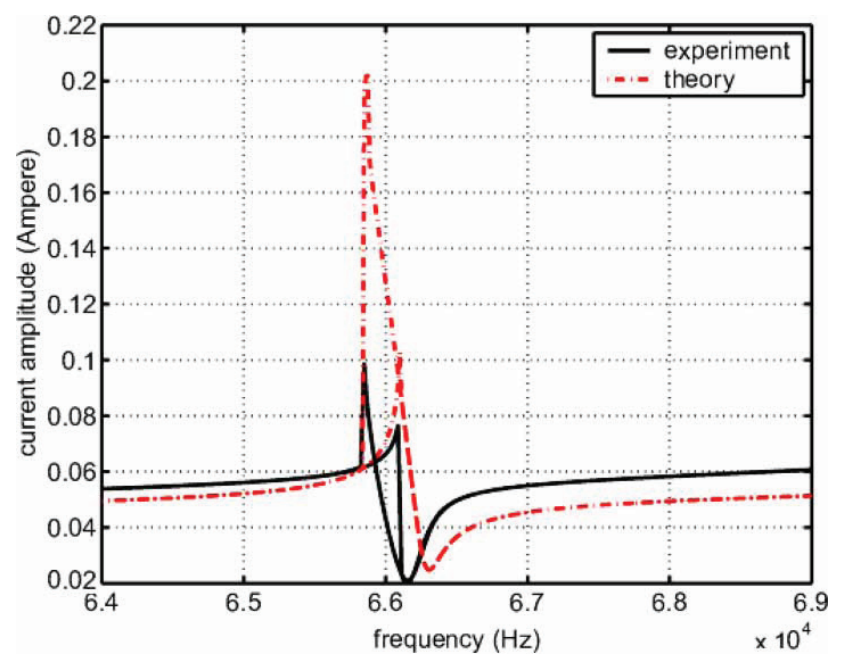

Fig. 12 Current response of the PIC 181 plate for the applied sinusoidal electric field of $7.5 \mathrm{~V} / \mathrm{mm}$ peak amplitude (second in-plane mode) have been presented here and compared with those of experiment. Figures 5 and 6 show the variation of displacement and current amplitudes with frequency at the electric field amplitude of $7.5 \mathrm{~V} / \mathrm{mm}$, and Figs 7 and 8 show the same at the electric field amplitude of $0.75 \mathrm{~V} / \mathrm{mm}$. Results have been shown for both sweepup and sweep-down modes of frequency response. As this piezoceramic material has a low damping property, the jump phenomenon can be clearly observed in Figs 5 and 6 respectively for both displacement and current responses of the plate. Again, the nonlinear behaviour (i.e. jump phenomenon) is dominant at higher electric field amplitudes, whereas at the very

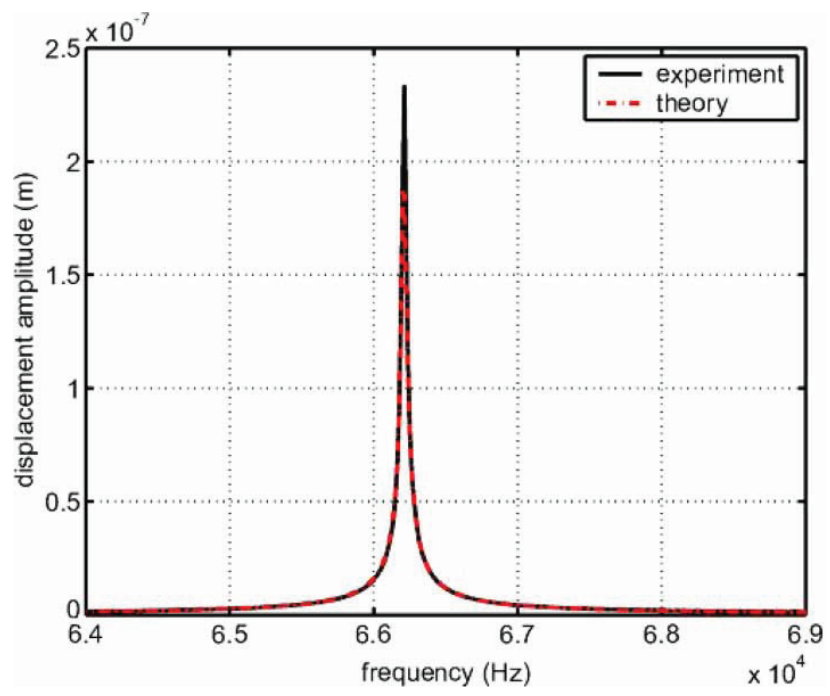

Fig. 13 Displacement response of the PIC 181 plate for the applied sinusoidal electric field of $0.75 \mathrm{~V} / \mathrm{mm}$ peak amplitude (second in-plane mode)

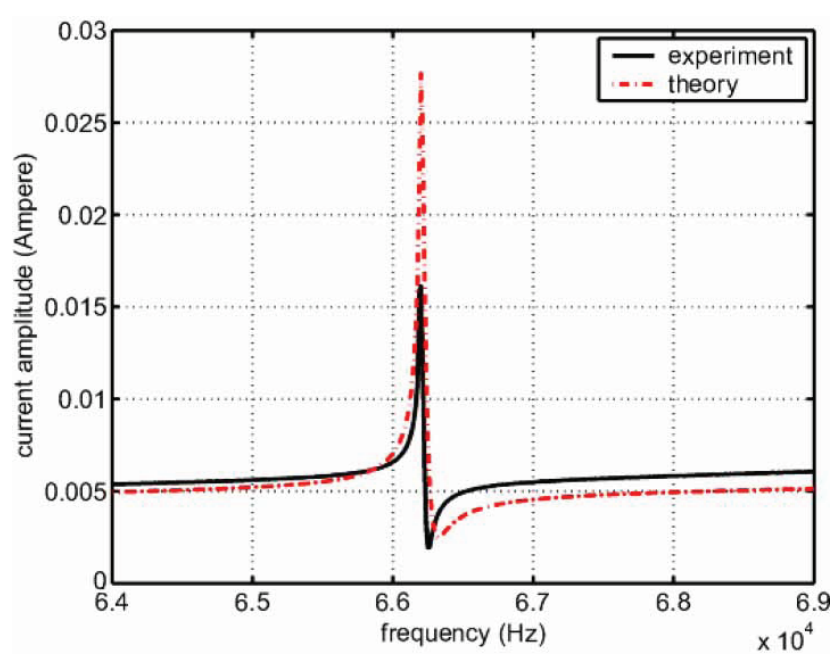

Fig. 14 Current response of the PIC 181 plate for the applied sinusoidal electric field of $0.75 \mathrm{~V} / \mathrm{mm}$ peak amplitude (second in-plane mode) 


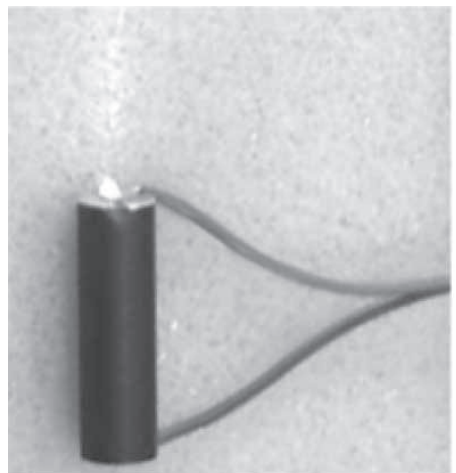

(a)

Fig. 15 (a) Piezoceramic cylinder excited in the longitudinal mode with electric field applied across the length and the vibration amplitude measured using laser vibrometer; (b) boundary conditions used for the axisymmetric modes of the cylinder

small values of applied electric field, the responses of both sweep-up and sweep-down modes are same and hence the behaviour is almost linear.

The other non-linearity observed in the frequency response is the dependence of the resonance frequency on applied electric field amplitude. The linear resonance frequency for the first in-plane mode of the plate is $23.65 \mathrm{kHz}$ as can be seen from frequencies corresponding to maximum amplitudes of Figs 7 and 8 where it has linear response. However, the frequency corresponding to maximum amplitude is different for sweep-up and sweep-down modes (Figs 5 and 6). This frequency is also less compared with the linear natural frequency, which corresponds to softening phenomenon in the piezoceramic and as the applied electric field increases, this frequency decreases, and hence for this type of non-linearity, the resonance frequency is dependent upon the amplitude of excitation. It can also be observed that the closed form solution has been able to represent the different experimental responses quite satisfactorily.

\subsection{Prediction of non-linear response of the PIC 181 rectangular plate in the second in-plane mode}

Experiments have also been carried on the same PIC 181 rectangular plate with electric field excitation near its second in-plane longitudinal vibration mode (the deformed mode-shape is shown in Fig. 9). The linear natural frequency corresponds to $66.2 \mathrm{kHz}$. For the analytical solution near the second in-plane mode, the wave numbers $\lambda_{1}, \lambda_{2}$, and $\lambda_{3}$ are taken as $3 \pi / l$ (the corresponding three-dimensional shape functions, i.e. $U(x, y, z), V(x, y, z)$, and $W(x, y, z)$, are plotted in Figs 10 (a) to (c), respectively, as contour plots on the original geometry) and hence the non-linear displacement and current responses are obtained using the same solution strategy as discussed for the first inplane longitudinal vibration mode. Results for applied electric fields of 7.5 and $0.75 \mathrm{~V} / \mathrm{mm}$ are discussed here. Figures 11 and 12 show the comparison of theoretical and experimental displacement and current responses, respectively, for the PIC 181 plate when excited near the second in-plane longitudinal vibration mode with an electric field of $7.5 \mathrm{~V} / \mathrm{mm}$. Experiment and analyses were carried out for both sweep-up and sweep-down frequency modes of excitation and at this applied electric field, the nonlinearities such as jump phenomena and dependence of resonance frequency on applied electric field are clearly observed. The responses at an electric field of $0.75 \mathrm{~V} / \mathrm{mm}$ is almost linear for both displacement

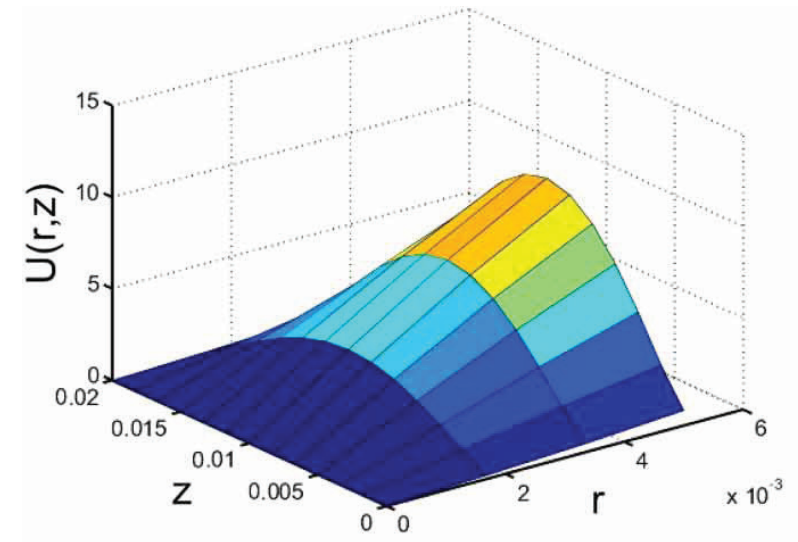

(a)

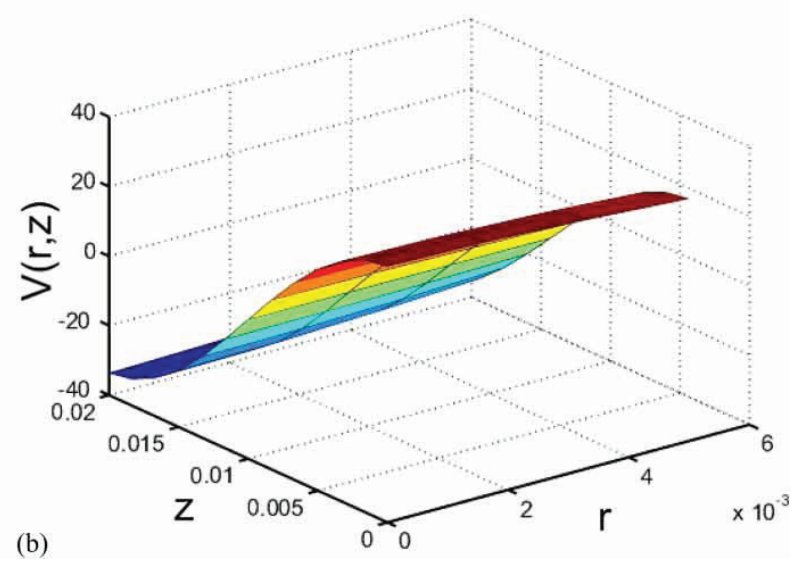

(b)

Fig. 16 Plot of (a) radial and (b) longitudinal displacements corresponding to the eigenvector of the first axisymmetric longitudinal mode shape of the piezocylinder 
and current responses (Figs 13 and 14). The comparison between experimental and theoretical results is satisfactory.

\subsection{Prediction of non-linear response of the PIC 181 cylinders ( 10 and $25 \mathrm{~mm}$ diameter) in the first longitudinal mode}

After demonstrating the prediction capability of the solution technique proposed in this work for the nonlinear response of piezoceramic materials with the help of rectangular piezoplates, the authors intend to demonstrate the same for another specimen geometry (i.e. a solid piezocylinder). They have carried out

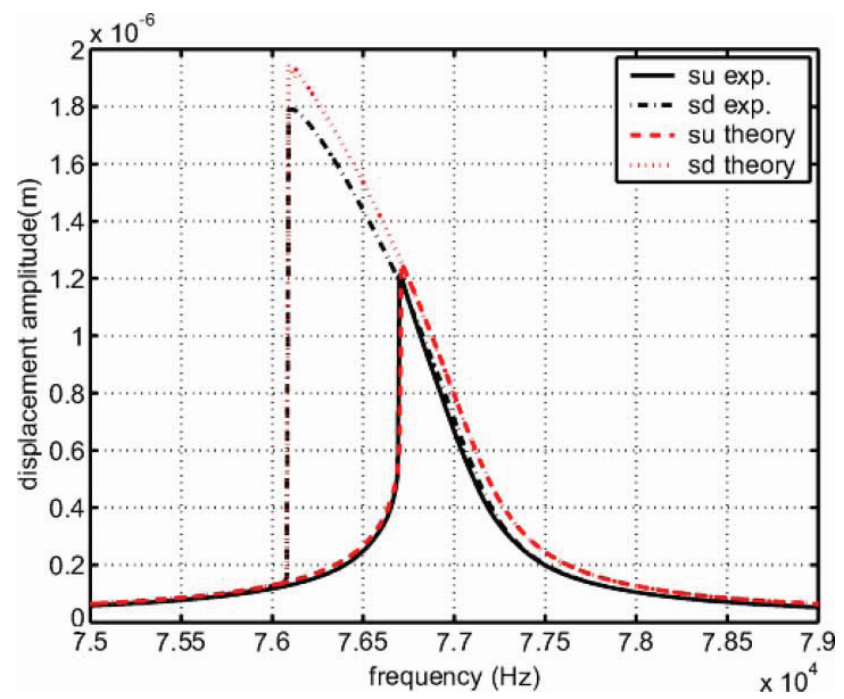

Fig. 17 Displacement response of PIC 181 cylinder (10 $\mathrm{mm}$ diameter) for the applied sinusoidal electric potential of $30 \mathrm{~V}$ peak amplitude

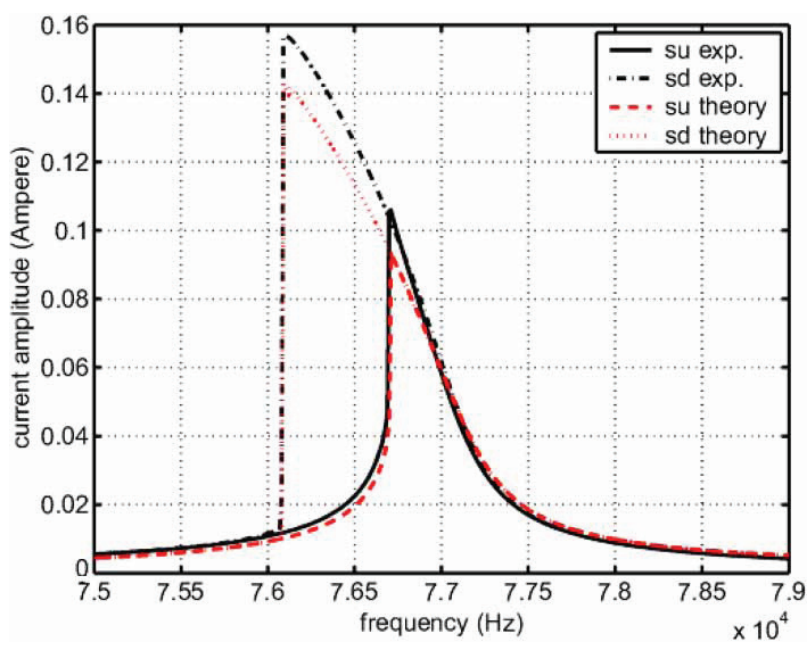

Fig. 18 Current response of PIC 181 cylinder $(10 \mathrm{~mm}$ diameter) for the applied sinusoidal electric potential of $30 \mathrm{~V}$ peak amplitude experiments on piezocylinders of PIC 181 (of $20 \mathrm{~mm}$ length and diameters ranging from 6 to $25 \mathrm{~mm}$ ). The electric potential is applied across the length of the piezocylinder as shown in Fig. 15(a). For the analysis, the authors consider the axisymmetric boundary conditions (Fig. 15(b)) as the modes under consideration are axisymmetric. The radial and longitudinal co-ordinates are denoted as $r$ and $z$ and the corresponding displacement are $U(r, z)$ and $V(r, z)$, respectively. In order to derive the mode shape function corresponding to the first longitudinal vibration mode of the cylinder numerical simulation (i.e. finite-element method) is used. The finite-element linear shape functions corresponding to this mode are plotted in Fig. 16. Once the shape functions are

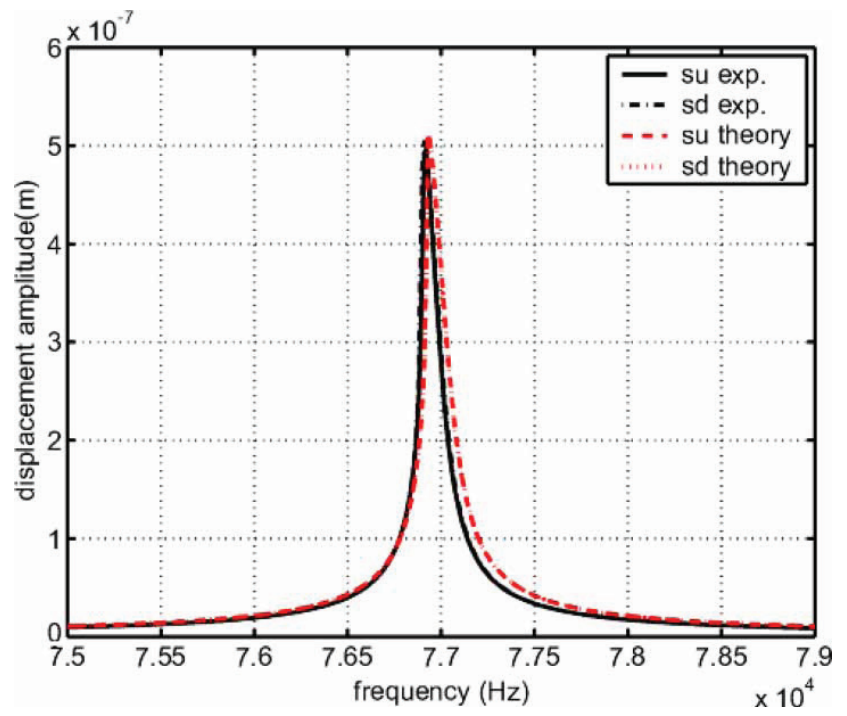

Fig. 19 Displacement response of PIC 181 cylinder (10 $\mathrm{mm}$ diameter) for the applied sinusoidal electric potential of $5 \mathrm{~V}$ peak amplitude

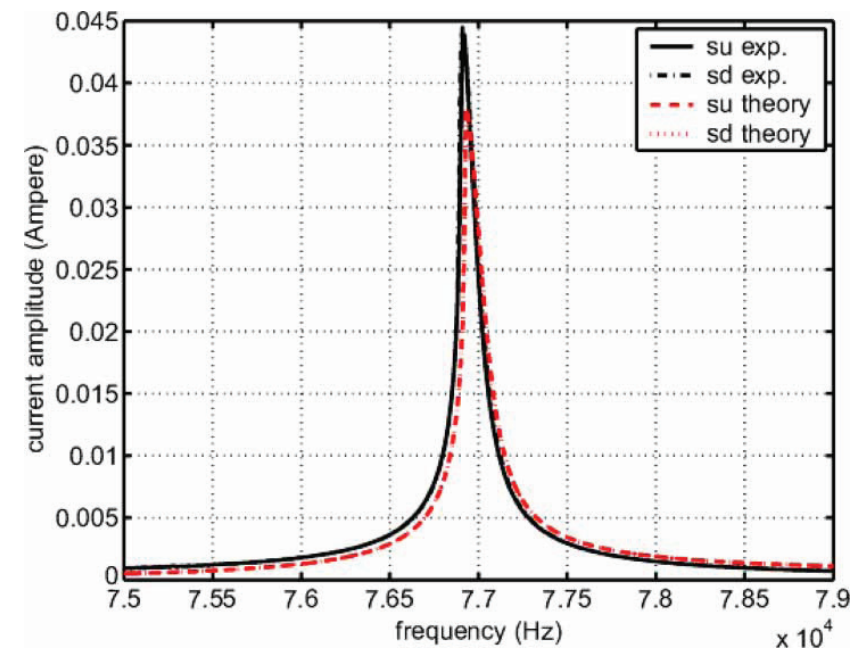

Fig. 20 Current response of PIC 181 cylinder $(10 \mathrm{~mm}$ diameter) for the applied sinusoidal electric potential of $5 \mathrm{~V}$ peak amplitude 
obtained, the procedure to obtain the displacement and current response is same as discussed for the plate geometry. The piezocylinders have been excited with sinusoidal electric fields of different amplitudes (in both sweep-up and sweep-down frequency modes). The amplitude of sinusoidal electric potential across the length ranges from 5 to $30 \mathrm{~V}$.

The results for displacement amplitude (at the end of the cylinder which is measured with laser vibrometer as shown in Fig. 15(a)) and current responses at 5 and $30 \mathrm{~V}$ of applied electric potential are presented and compared with those of the experiment. Figures 17 and 18 show the non-linear displacement and current responses for the sweep-up and sweep-down modes

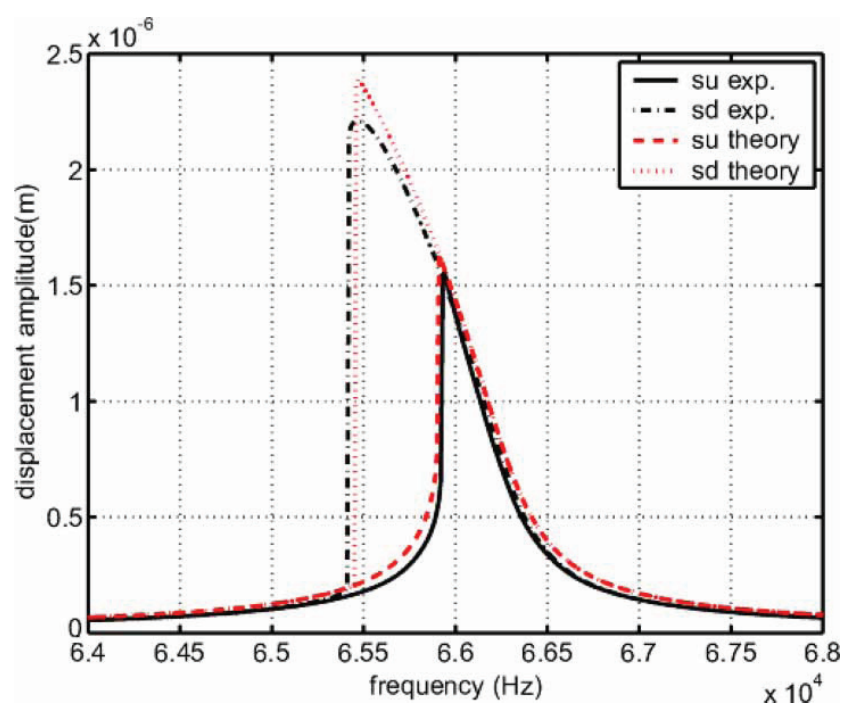

Fig. 21 Displacement response of PIC 181 cylinder (25 $\mathrm{mm}$ diameter) for the applied sinusoidal electric potential of $30 \mathrm{~V}$ peak amplitude

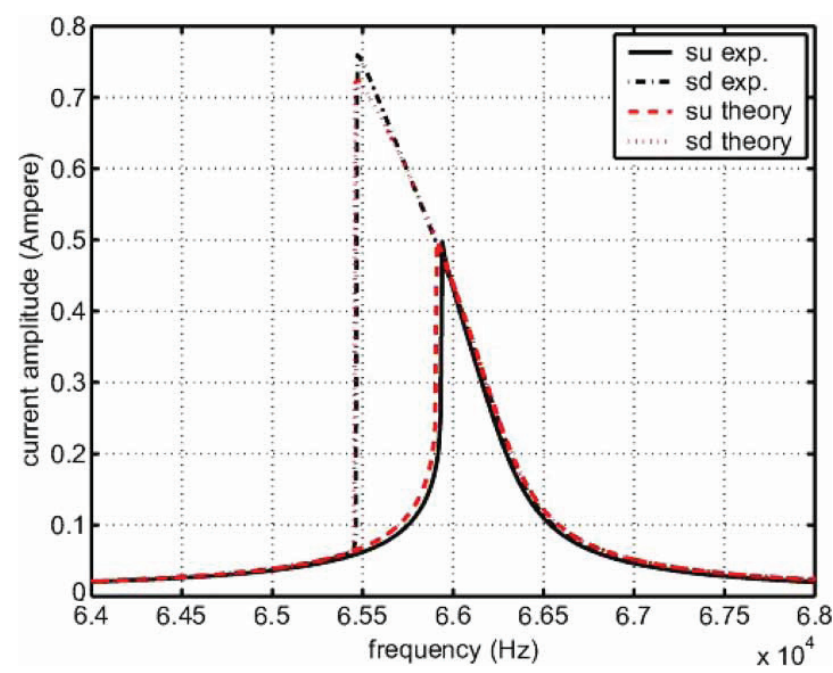

Fig. 22 Current response of PIC 181 cylinder $(25 \mathrm{~mm}$ diameter) for the applied sinusoidal electric potential of $30 \mathrm{~V}$ peak amplitude of frequency response of the cylinder of $10 \mathrm{~mm}$ diameter. It can be observed that non-linearities similar to those of the plate geometry are also observed here and predicted results compare very well with those of experiment. For $5 \mathrm{~V}$ electric potential amplitude, the response of the piezocylinder is linear as can be seen from Figs 19 and 20. The results of non-linear responses of the piezocylinder of $25 \mathrm{~mm}$ diameter are shown in Figs 21 and 22 corresponding to $30 \mathrm{~V}$ of applied electric potential, whereas at $5 \mathrm{~V}$ of applied electric potential, the responses are linear (Figs 23 and 24). Again, the predictions compare very well with those of experiment for the piezocylinder with different diameters.

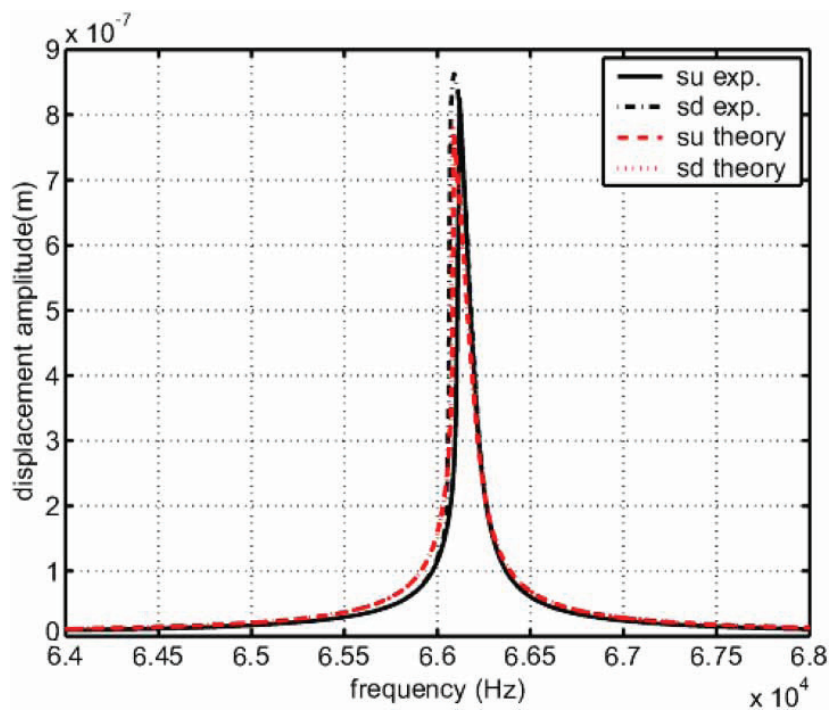

Fig. 23 Displacement response of PIC 181 cylinder (25 mm diameter) for the applied sinusoidal electric potential of $5 \mathrm{~V}$ peak amplitude

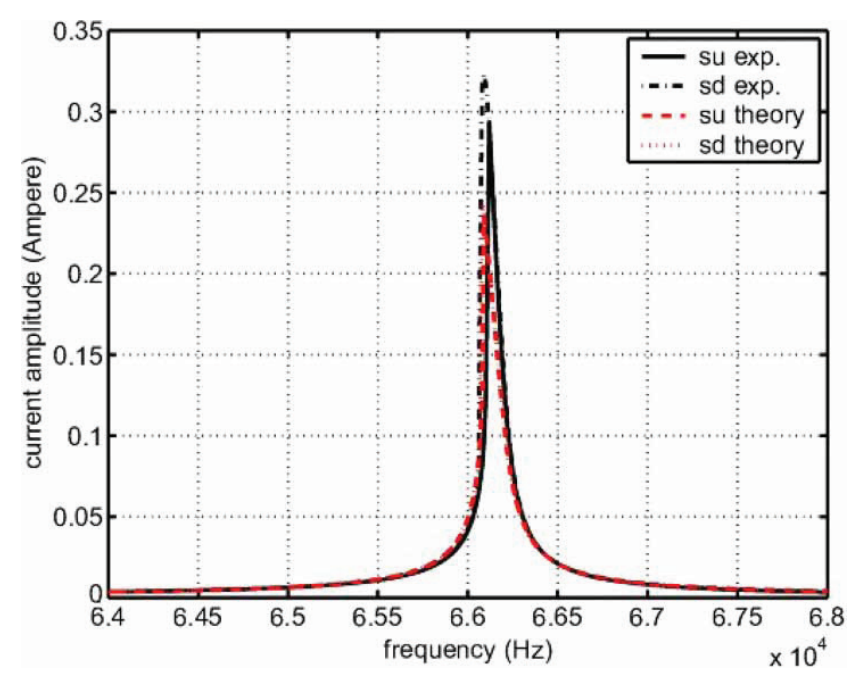

Fig. 24 Current response of PIC 181 cylinder $(25 \mathrm{~mm}$ diameter) for the applied sinusoidal electric potential of $5 \mathrm{~V}$ peak amplitude 
The linear natural frequency of the piezocylinders depends upon their diameter (for the same length as stiffness and inertia properties change) and hence the linear natural frequency of the piezocylinder with $10 \mathrm{~mm}$ diameter is $66.1 \mathrm{kHz}$ whereas it is $76.91 \mathrm{kHz}$ for the piezocylinder with $25 \mathrm{~mm}$ diameter. When they are excited with an electric potential of $30 \mathrm{~V}$, it can be observed (Figs 17 and 21) that the frequencies corresponding to resonance peaks in both sweep-up and sweep-down modes decrease and the extent of decrease depends upon the cylinder diameter. For example, the sweep-down resonance peak for the cylinder with $10 \mathrm{~mm}$ diameter (Fig. 17) decreased to $65.4 \mathrm{kHz}$ whereas the same peak for the cylinder with $25 \mathrm{~mm}$ diameter (Fig. 21) decreased to $65.08 \mathrm{kHz}$. These results demonstrate the fact that the non-linear response depends upon the geometrical dimensions of piezomaterial and the model described here is able to predict both the displacement and current responses satisfactorily for the piezomaterial with different geometries (i.e. plate and cylinders with different diameters).

\section{CONCLUSIONS}

Piezoelectric continuum exhibits different types of non-linearities under weak electric fields (when the system is operating near resonance frequency) such as jump phenomena, dependence of resonance frequency on vibration amplitude, etc. In this work, a generalized non-linear electric enthalpy density function incorporating higher-order non-linear terms (quadratic and cubic) in the energy expression of the coupled piezoelectric medium has been formulated. Damping has been formulated using proportional damping method. This non-linear electric enthalpy density function, as well as the virtual work due to proportional damping, has been used in the Hamilton principle to get the variational form of the equations of motion. This form has been discretized using Rayleigh-Ritz method.

The shape function of the linear eigenvalue problem corresponding to the desired mode of vibration of the rectangular piezoelectric plate has been obtained by solving the governing equations of motion along with the boundary conditions for the three-dimensional domain. These shape functions are used in the Rayleigh-Ritz method to get the non-linear equation. The non-linear equation is expanded using the perturbation technique of Lindstedt and Poincaré. The solution for the vibration amplitude has been obtained finally as the solution of a polynomial equation where the coefficients are functions of the material properties. Experiments have been conducted on a rectangular plate geometry of PIC 181 piezoceramic in both first and second in-plane modes. The closed form solutions obtained from three-dimensional analysis have been compared with the experimental results. It is observed that the closed form solutions were able to reproduce the experimental non-linear responses quite satisfactorily.

Experiments have also been conducted on piezocylinders of different diameters of PIC 181 with electric field excitations along the length of the cylinder corresponding to the axisymmetric modes. Mode-shapes of the cylinder for the Rayleigh-Ritz procedure have been obtained numerically using finite-element method. Following the same procedure as the piezoplate, the non-linear displacement and current responses of the piezocylinder have been predicted for different amplitudes of electric field excitations. The predicted results have been compared with experiments and it was observed that the method could predict satisfactory responses for different types of geometries. These closed form solutions should form a basis to validate the finite-element formulations for this nonlinear problem as well as determination of material parameters using optimization procedure.

\section{REFERENCES}

1 Crawley, E. F. Intelligent structures for aerospace: a technology overview and assessment. AIAA J., 1994, 32(8), 1689-1699.

2 Viswamurthy, S. R., Rao, A. K., and Ganguli, R. Dynamic hysteresis of piezoceramic stack actuators used in helicopter vibration control: experiments and simulations. Smart Mater. Struct., 2007, 16, 1109-1119.

3 Viswamurthy, S. R. and Ganguli, R. Modeling and analysis of piezoceramic actuator hysteresis for helicopter vibration control. Sens. Actuators A, Phys., 2007, 35(2), 801-810.

4 Hwang, S. C. and McMeeking, R. M. A finite element model of ferroelectric polycrystals. Ferroelectrics, 1998, 211, 177-194.

5 Thakkar, D. and Ganguli, R. Induced shear actuation of helicopter rotor blade for active twist control. ThinWalled Struct., 2007, 45(1), 111-121.

6 Thakkar, D. and Ganguli, R. Use of single crystal and soft piezoceramics for alleviation of flow separation induced vibration in smart helicopter rotor. Smart Mater. Struct., 2006, 15, 331-341.

7 Thakkar, D. and Ganguli, R. Helicopter vibration reduction in forward flight with induced shear based piezoceramic actuation. Smart Mater. Struct., 2004, 13(3), 599-608.

8 Pagano, N. J. Exact solutions for rectangular bidirectional composites and sandwich plates. J. Compos. Mater., 1970, 4(1), 20-34.

9 Ray, M. C., Rao, K. M., and Samanta, B. Exact analysis of coupled electro-elastic behaviour of a piezoelectric plate under cylindrical bending. Comput. Struct., 1992, 45(4), 667-677. 
10 Ray, M. C., Rao, K. M., and Samanta, B. Exact solution for static analysis of an intelligent structure under cylindrical bending. Comput. Struct., 1993, 47(6), 1031-1042.

11 Ray, M. C., Bhattacharya, R., and Samanta, B. Exact solutions for static analysis of intelligent structures. AIAA J., 1993, 31 (1), 1684-1691.

12 Heyliger, P. and Brooks, S. Exact solutions for laminated piezoelectric plates in cylindrical bending. Trans. ASME, J. Appl. Mech., 1996, 63(4), 903-910.

13 Xu, K. M., Noor, A. K., and Tang, Y. Three-dimensional solutions for coupled thermoelectroelastic response of multilayered plates. Comput. Methods Appl. Mech. Eng., 1995, 126(3-4), 355-371.

14 Dube, G. P., Kapuria, S., and Dumir, P. C. Exact piezothermoelastic solution of simply supported orthotropic flat panel in cylindrical bending. Int. J. Mech. Sci., 1996, 38(3), 1161-1177.

15 Ray, M. C., Bhattacharya, R., and Samanta, B. Exact solutions for dynamic analysis of composite plates with distributed piezoelectric layers. Comput. Struct., 1998, 66(6), 737-743.

16 Ray, M. C. Closed-form solution for optimal control of laminated plate. Comput. Struct., 1998, 69(2), 283-290.

17 Wolf, K. and Gottlieb, O. Nonlinear dynamics of a cantilever beam actuated by piezoelectric layers in symmetric and asymmetric configuration. Technical report ETR-2001-02, Faculty of Mechanical Engineering, Israel Institute of Technology, Israel, 2001.

18 Beige, H. and Schmidt, G. Electromechanical resonances for investigating linear and nonlinear properties of dielectrics. Ferroelectrics, 1982, 41(1), 39-49.

19 von Wagner, $\mathbf{U}$. and Hagedorn, P. Piezo-beam-systems subjected to weak electric field: experiment and modelling of nonlinearities. J. Sound Vibr., 2002, 256(5), 861-872.

20 von Wagner, $\mathbf{U}$. Non-linear longitudinal vibrations of piezoceramics excited by weak electric fields. Int. J. Non-Linear Mech., 2003, 38(4), 565-574.

21 Neumann, N. Nichtlineare Effekte bei Längsschwingungen axial polarisierter piezokeramischer Staabe: Experimentelle Untersuchungen und Parameteridentifikation. Diplomarbeit, Institut für Mechanik, Technische Universität Darmstadt, 2002.

22 Samal, M. K., Seshu, P., Parashar, S. K., von Wagner, U., Hagedorn, P., Dutta, B. K., and Kushwaha, H. S. Nonlinear behaviour of piezoceramics under weak electric fields, part 1:3-D finite element formulation. Int. J. Solids Struct., 2006, 43(6), 1422-1436.

23 Samal, M. K., Seshu, P., Parashar, S. K., von Wagner, U., Hagedorn, P., Dutta, B. K., and Kushwaha, H. S. Nonlinear behaviour of piezoceramics under weak electric fields, part 2: numerical results and validation with experiments. Int. J. Solids Struct., 2006, 43(6), 1437-1458.

24 Samal, M. K., Seshu, P., and Dutta, B.K. An analytical formulation in $3 \mathrm{D}$ domain for the nonlinear response of piezoelectric slabs under weak electric fields. Int. J. Solids Struct., 2007, 44, 4656-4672.

25 Usher, T. and Sim, A. Nonlinear dynamics of piezoelectric high displacement actuators in cantilever mode. J. Appl. Phys., 2005, 98(6), 064102, pp. 1-7.

26 Wang, Q.-M., Zhang, Q., Xu, B., Liu, R., and Cross, L. E. Nonlinear piezoelectric behavior of ceramic bending mode under strong electric fields. J. Appl. Phys., 1999, 86(6), 3352-3360.

27 IEEE Standards on Piezoelectricity. ANSI/IEEE Standard, The Institute of Electrical and Electronic Engineers, New York, 1988.

28 Maugin, G. A. Nonlinear electromechanical effects and applications, 1985 (World Scientific Publishing Company, Singapore).

29 Nayfeh, A. H. and Mook, D. Nonlinear oscillations, 1979 (Willey publications, New York).

30 Parashar, S. K. and von Wagner, U. Nonlinear longitudinal vibrations of transversally polarized piezoceramics: experiments and modeling. Nonlinear Dyn., 2004, 37(1), 51-73.

31 Samal, M. K. Modelling of nonlinear behaviour of piezoelectric materials under weak electric fields. MSc Thesis, Department of Mechanical Engineering, Indian Institute of Technology, Bombay, 2003.

\section{APPENDIX 1}

\section{Boundary conditions for equation (14)}

The boundary conditions are derived as

$$
\begin{aligned}
& \int_{-h / 2}^{+h / 2} \int_{-b / 2}^{+b / 2}\left[c_{11} \frac{\partial u}{\partial x}+c_{12} \frac{\partial v}{\partial y}+c_{13} \frac{\partial w}{\partial z}\right. \\
& \left.+E_{z}\left(d_{31} c_{11}+d_{32} c_{12}++d_{33} c_{13}\right)\right]_{x= \pm l / 2} \mathrm{~d} y \mathrm{~d} z=0 \\
& \int_{-h / 2}^{+h / 2} \int_{-l / 2}^{+l / 2}\left[c_{21} \frac{\partial u}{\partial x}+c_{22} \frac{\partial v}{\partial y}++c_{23} \frac{\partial w}{\partial z}\right. \\
& \left.+E_{z}\left(d_{31} c_{21}+d_{32} c_{22}++d_{33} c_{23}\right)\right]_{y= \pm b / 2} \mathrm{~d} x \mathrm{~d} z=0 \\
& \int_{-b / 2}^{+b / 2} \int_{-l / 2}^{+l / 2}\left[c_{31} \frac{\partial u}{\partial x}+c_{32} \frac{\partial v}{\partial y}++c_{33} \frac{\partial w}{\partial z}\right. \\
& \left.+E_{z}\left(d_{31} c_{31}+d_{32} c_{32}++d_{33} c_{33}\right)\right]_{z= \pm h / 2} \mathrm{~d} x \mathrm{~d} y=0 \\
& \int_{-h / 2}^{+h / 2} \int_{-b / 2}^{+b / 2}\left[c_{66}\left(\frac{\partial u}{\partial y}+\frac{\partial v}{\partial x}\right)\right]_{x= \pm l / 2} \mathrm{~d} y \mathrm{~d} z=0 \\
& \int_{-h / 2}^{+h / 2} \int_{-l / 2}^{+l / 2}\left[c_{66}\left(\frac{\partial u}{\partial y}+\frac{\partial v}{\partial x}\right)\right]_{y= \pm b / 2} \mathrm{~d} x \mathrm{~d} z=0 \\
& \int_{-h / 2}^{+h / 2} \int_{-h / 2}^{+l / 2}\left[c_{-l / 2}^{+h / 2} \int_{-l / 2}^{+b / 2}\left[c_{44}\left(\frac{\partial v}{\partial z}+\frac{\partial w}{\partial y}\right)\right]_{y= \pm b / 2} \mathrm{~d} x \mathrm{~d} z=0\right. \\
& \left.\int_{-l / 2}^{+h / 2} \int_{55}^{+l / 2}\left[\frac{\partial u}{\partial z}+\frac{\partial w}{\partial x}\right)\right]_{x= \pm l / 2} \mathrm{~d} y \mathrm{~d} z=0 \\
& \int_{z= \pm h / 2} \mathrm{~d} x \mathrm{~d} y=0 \\
& \left.\int_{-h}\left(\frac{\partial u}{\partial z}+\frac{\partial w}{\partial x}\right)\right]_{z= \pm h / 2} \mathrm{~d} x \mathrm{~d} y=0
\end{aligned}
$$




\section{APPENDIX 2}

\section{Coefficients of equation (21)}

$$
\begin{array}{r}
m_{k}=\rho \int_{-h / 2}^{+h / 2} \int_{-b / 2}^{+b / 2} \int_{-l / 2}^{+l / 2}\left[U^{2}(x, y, z)+V^{2}(x, y, z)\right. \\
\left.+W^{2}(x, y, z)\right] \mathrm{d} x \mathrm{~d} y \mathrm{~d} z
\end{array}
$$$$
c_{k}=\int_{-h / 2}^{+h / 2} \int_{-b / 2}^{+b / 2} \int_{-l / 2}^{+l / 2}\left[\left\{\boldsymbol{S}^{*}\right\}^{\mathrm{T}}[\mathbf{c}]\left\{\boldsymbol{S}^{*}\right\}\right] \mathrm{d} x \mathrm{~d} y \mathrm{~d} z
$$$$
B_{k}=\int_{-h / 2}^{+h / 2} \int_{-b / 2}^{+b / 2} \int_{-l / 2}^{+l / 2}\left[\left\{\boldsymbol{S}^{*}\right\}^{\mathrm{T}}\left[\mathbf{c}_{1}\right]\left\{\boldsymbol{S}^{* 2}\right\}\right] \mathrm{d} x \mathrm{~d} y \mathrm{~d} z
$$$$
\varepsilon_{k}=\int_{-h / 2}^{+h / 2} \int_{-b / 2}^{+b / 2} \int_{-l / 2}^{+l / 2}\left[\left\{\boldsymbol{S}^{* 2}\right\}^{\mathrm{T}}\left[\mathbf{c}_{21}\right]\left\{\boldsymbol{S}^{* 2}\right\}\right.
$$$$
\left.+\left\{\boldsymbol{S}^{*}\right\}^{\mathrm{T}}\left[\mathbf{c}_{22}\right]\left\{\boldsymbol{S}^{* 3}\right\}\right] \mathrm{d} x \mathrm{~d} y \mathrm{~d} z
$$

$$
\begin{aligned}
& f_{k}^{(1)}=\int_{-h / 2}^{+h / 2} \int_{-b / 2}^{+b / 2} \int_{-l / 2}^{+l / 2}\left[\left\{\boldsymbol{E}^{*}\right\}^{\mathrm{T}}[\mathbf{d}][\mathbf{c}]\left\{\boldsymbol{S}^{*}\right\}\right] \mathrm{d} x \mathrm{~d} y \mathrm{~d} z \\
& f_{k}^{(2)}=\int_{-h / 2}^{+h / 2} \int_{-b / 2}^{+b / 2} \int_{-l / 2}^{+l / 2}\left[\left\{\boldsymbol{E}^{*}\right\}^{\mathrm{T}}\left[\boldsymbol{\gamma}_{11}\right]\left\{\boldsymbol{S}^{* 2}\right\}\right] \mathrm{d} x \mathrm{~d} y \mathrm{~d} z \\
& f_{k}^{(3)}=\int_{-h / 2}^{+h / 2} \int_{-b / 2}^{+b / 2} \int_{-l / 2}^{+l / 2}\left[\left\{\boldsymbol{E}^{*}\right\}^{\mathrm{T}}\left[\boldsymbol{\gamma}_{21}\right]\left\{\boldsymbol{S}^{* 3}\right\}\right] \mathrm{d} x \mathrm{~d} y \mathrm{~d} z \\
& f_{k}^{(4)}=\int_{-h / 2}^{+h / 2} \int_{-b / 2}^{+b / 2} \int_{-l / 2}^{+l / 2}\left[\frac{1}{2}\left\{\boldsymbol{E}^{* 2}\right\}^{\mathrm{T}}\left[\boldsymbol{\gamma}_{12}\right]\left\{\boldsymbol{S}^{*}\right\}\right] \mathrm{d} x \mathrm{~d} y \mathrm{~d} z \\
& f_{k}^{(5)}=\int_{-h / 2}^{+h / 2} \int_{-b / 2}^{+b / 2} \int_{-l / 2}^{+l / 2}\left[\left\{\boldsymbol{E}^{* 2}\right\}^{\mathrm{T}}\left[\boldsymbol{\gamma}_{22}\right]\left\{\boldsymbol{S}^{* 2}\right\}\right] \mathrm{d} x \mathrm{~d} y \mathrm{~d} z \\
& f_{k}^{(6)}=\int_{-h / 2}^{+h / 2} \int_{-b / 2}^{+b / 2} \int_{-l / 2}^{+l / 2}\left[\frac{1}{3}\left\{\boldsymbol{E}^{* 3}\right\}^{\mathrm{T}}\left[\boldsymbol{\gamma}_{23}\right]\left\{\boldsymbol{S}^{*}\right\}\right] \mathrm{d} x \mathrm{~d} y \mathrm{~d} z
\end{aligned}
$$

where

$$
\begin{aligned}
& \left\{\boldsymbol{S}^{*}\right\}=\left\{\begin{array}{c}
\frac{\partial U(x, y, z)}{\partial x} \\
\frac{\partial V(x, y, z)}{\partial y} \\
\frac{\partial W(x, y, z)}{\partial z} \\
\frac{\partial W(x, y, z)}{\partial y}+\frac{\partial V(x, y, z)}{\partial z} \\
\frac{\partial W(x, y, z)}{\partial x}+\frac{\partial U(x, y, z)}{\partial z} \\
\frac{\partial V(x, y, z)}{\partial x}+\frac{\partial U(x, y, z)}{\partial y}
\end{array}\right\} ; \quad\left\{\boldsymbol{S}^{* 2}\right\}=\left\{\begin{array}{c}
\left(\frac{\partial U(x, y, z)}{\partial x}\right)^{2} \\
\left(\frac{\partial V(x, y, z)}{\partial y}\right)^{2} \\
\left(\frac{\partial W(x, y, z)}{\partial z}\right)^{2} \\
\left(\frac{\partial W(x, y, z)}{\partial y}+\frac{\partial V(x, y, z)}{\partial z}\right)^{2} \\
\left(\frac{\partial W(x, y, z)}{\partial x}+\frac{\partial U(x, y, z)}{\partial z}\right)^{2} \\
\left(\frac{\partial V(x, y, z)}{\partial x}+\frac{\partial U(x, y, z)}{\partial y}\right)^{2}
\end{array}\right\}
\end{aligned}
$$

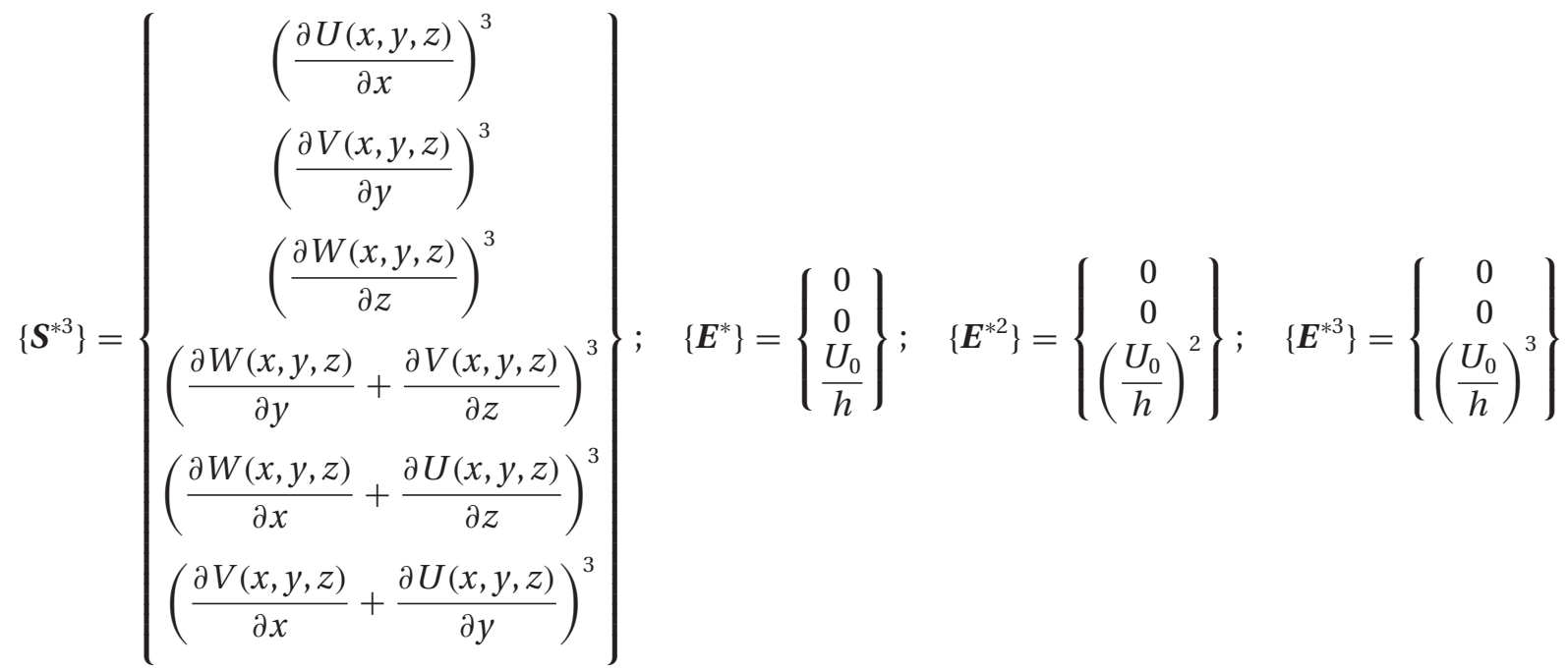




\section{APPENDIX 3}

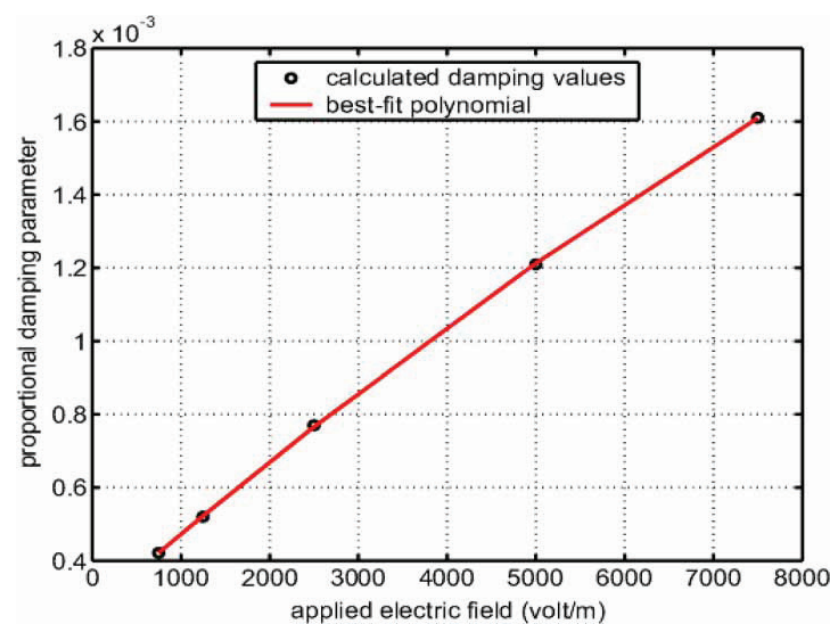

Fig. 25 Variation of stiffness proportional damping constant with the applied electric field for the piezoceramic PIC 181

The material properties of PIC 181 are as follows

(Fig. 25)

$$
\begin{aligned}
{\left[\mathbf{S}^{\mathrm{E}}\right] } & =\left[\begin{array}{cccccc}
1.1600 & -0.4070 & -0.4996 & 0 & 0 & 0 \\
-0.4070 & 1.1750 & -0.4996 & 0 & 0 & 0 \\
-0.4996 & -0.4996 & 1.4110 & 0 & 0 & 0 \\
0 & 0 & 0 & 3.5330 & 0 & 0 \\
0 & 0 & 0 & 0 & 3.5330 & 0 \\
0 & 0 & 0 & 0 & 0 & 3.1640
\end{array}\right] \times 10^{-11} \mathrm{~m}^{2} / \mathrm{N} \\
{[\mathbf{d}]=} & {\left[\begin{array}{cccccc}
0 & 0 & 0 & 0 & 3.89 & 0 \\
0 & 0 & 0 & 3.89 & 0 & 0 \\
-1.148 & -1.148 & 2.53 & 0 & 0 & 0
\end{array}\right] 10^{-10} \mathrm{~m} / \mathrm{V} } \\
{\left[\mathbf{C}_{21}\right] } & =-\left[\begin{array}{cccccc}
4.7000 & 2.7914 & 2.6525 & 0 & 0 & 0 \\
2.7914 & 4.7000 & 2.6525 & 0 & 0 & 0 \\
2.6525 & 2.6525 & 3.9978 & 0 & 0 & 0 \\
0 & 0 & 0 & 0.8465 & 0 & 0 \\
0 & 0 & 0 & 0 & 0.8465 & 0 \\
0 & 0 & 0 & 0 & 0 & 0.9452
\end{array}\right] \times 10^{16} \mathrm{~N} / \mathrm{m}^{2}
\end{aligned}
$$

